\title{
Effect of Pivot Point on Aerodynamic Force and Vortical Structure of Pitching Flat Plate Wings
}

\author{
Huai-Te $\mathrm{Yu}^{1}$ and Luis P. Bernal ${ }^{2}$ \\ University of Michigan, Ann Arbor, MI, 48109
}

\begin{abstract}
This paper reports aerodynamic forces and 2D PIV velocity measurements on a rectangular wing with an effective aspect ratio 4 during a pitch ramp-hold wing motion at reduced frequencies from 0.022 to 0.39 . The parameter space in terms of the Stokes number and the Reynolds number is used to correlate pitch rate and free stream velocity effects. The maximum angle of attack is $\mathbf{4 5}$ degrees and pivot locations, such as leading edge pivot, midchord pivot, and trailing edge pivot, are considered. The evolution of aerodynamic forces as functions of convective time, pitching time, and angle of attack are discussed. Noncirculatory effects are observed for reduced frequency higher than 0.13. For leading edge pivot axis non-circulatory effects produce a positive force spike at the start of rotation and a negative spike at the end of rotation. The opposite is found for trailing edge pivot. For midchord pivot the non-circulatory force spikes are not present. Rotation rate effects are observed during the constant rate part of the motion for reduced frequency higher than 0.065. For leading edge pivot axis non-circulatory effects and rotation rate effects combine to produce very large lift and drag coefficients. Incipient vortex shedding is observed at high reduced frequency during the hold phase for convective times in the range 4-20. For reduced frequency lower than $\mathbf{0 . 0 2 2}$ the flow is quasi steady and lifting line theory provides good estimates of lift for angle of attack below the steady stall angle. Although significant leading edge suction was measured at the final steady state condition, projection of the normal force in the drag direction is the main contribution to the drag. PIV measurements show the flow topology for different pivot axes. For leading edge pivot axis a starting vortex is formed at the trailing edge which promotes development of the LEV and enhances aerodynamic forces. For mid-chord pivot axis starting vortices are formed at the leading and trailing edges. For trailing edge pivot axis the starting vortex is formed at the leading edge only. Formation of a starting vortex at the leading edge delays development of the LEV and inhibits aerodynamic force generation.
\end{abstract}

\section{Nomenclature}

$\begin{array}{ll}A R & =\text { aspect ratio (physical) } \\ A R_{\text {eff }} & =\text { effective aspect ratio, } 2 A R \\ a & =\text { free parameter } \\ C_{D} & =\text { drag coefficient } \\ C_{L} & =\text { lift coefficient } \\ c & =\text { wing chord length } \\ \text { Drag } & =\text { drag force } \\ e & =\text { relaxation parameter } \\ F_{x} & =X \text { component of force in force transducer frame } \\ F_{y} & =Y \text { component of force in force transducer frame } \\ f & =\text { frequency, } H z \\ f_{c} & =\text { convective frequency, } f t_{c} \\ f_{p} & =\text { pitching frequency, } f t_{p}\end{array}$

\footnotetext{
${ }^{1} \mathrm{PhD}$ candidate, Department of Aerospace Engineering, University of Michigan, htyu@ umich.edu, AIAA Student Member.

${ }^{2}$ Associate Professor, Department of Aerospace Engineering, University of Michigan, lpb@umich.edu, AIAA Member.
} 


$\begin{array}{ll}h & =\text { hold parameter } \\ k & =\text { reduced frequency, } k=\dot{\alpha}_{m} c / 2 U_{\infty} \\ L & =\text { wing wetted length } \\ \text { Lift } & =\text { lift force } \\ R e_{c} & =\text { convective Reynolds number, } c U_{\infty} / v \\ R e_{p} & =\text { pitching Stokes number, } \dot{\alpha} c^{2} / v \\ S & =\text { wing surface area } \\ s & =\text { start parameter } \\ t_{1} & =\text { time that wings start to pitch-up in an unsmoothing trace, } \Delta \mathrm{T}_{\mathrm{s}} \\ t_{2} & =\text { time that wings start to hold in an unsmoothing trace, } t_{l}+\Delta \mathrm{T}_{\mathrm{p}} \\ t_{3} & =\text { time that wings start to pitch-down in an unsmoothing trace, } t_{2}+\Delta \mathrm{T}_{\mathrm{h}} \\ t_{4} & =\text { time that wings stop at the origin in an unsmoothing trace, } t_{3}+\Delta \mathrm{T}_{\mathrm{p}} \\ t_{5} & =\text { time that the motion is complete, } t_{4}+\Delta \mathrm{T}_{\mathrm{e}} \\ t_{c} & =\text { convective time, } U / c \\ t_{p} & =\text { pitching time, } \alpha_{m} / \dot{\alpha}_{m} \\ U_{\infty} & =\text { free stream velocity } \\ \alpha & =\text { angle of attack } \\ \dot{\alpha}_{m} & =\text { maximum pitch rate } \\ \alpha_{m} & =\text { maximum angle of attack } \\ \Delta \mathrm{T}_{\mathrm{p}} & =\text { pitching duration }\end{array}$

\section{Introduction}

L eading edge and tip vortices are important features of the flow development of finite aspect ratio wings undergoing rapid pitch maneuvers. Recently, Granlund et al ${ }^{1}$ studied perching motions of 2D airfoils and noted the significant effects of pivot axis location on force development during the airfoil motion. Also, Ramesh et al ${ }^{2}$ report computations and flow visualization experiments of pitching $2 \mathrm{D}$ airfoils for several maximum angles of attack and pivot point locations and noted that pivot axis has a profound impact on the shape of the lift distribution for the same motion kinematics. The developments of circulatory and non-circulatory apparent mass effects are different, depending on pivot point location. How pivot location impacts non-circulatory effects, rotation rate effects, and the formation of LEV, TEV and tip vortices are not well understood. Most prior work reported in the literature was conducted at relatively small reduced frequency, up to a maximum value of $\mathrm{k} \sim 0.2$. The present paper reports recent experiments on the aerodynamics of rapidly pitching finite-aspect-ratio wings with varying pivot point location and at several reduced frequencies. Here we extend work by Baik $e t a l^{3}$ and $\mathrm{Yu} e t a l^{4}$ and consider a finite aspect ratio wing (effective aspect ratio 4) undergoing rapid rotation from $0^{\circ}$ to $45^{\circ}$ pitch angle and with reduced frequency up to 0.39. Aerodynamic force and flow evolution using 2D PIV measurements are reported. The main focus of this research is to extend earlier work to higher reduced frequency for a finite aspect ratio wing, and to explore the relationship between vortical flow development and aerodynamic force for different pivot axis locations. The correlations of rotation rate and non-circulatory apparent mass are also provided.

Harper and Flanigan ${ }^{5}$ consider the effect of pitch rate on the maximum lift coefficient of a model aircraft and showed an increase in maximum lift coefficient with pitch rate along the steady lift-vs.-angle-of-attack curve. More recent work on dynamic stall have documented that the increase in lift is associated with the formation of a leading edge vortex $(\mathrm{LEV})^{6}$. Currier and Fung ${ }^{7}$ investigated the onset of dynamic stall ( $k$ in the range from 0 to 0.19 ), and suggested that the benefit from dynamic stall is present only during the separation process and inviscid theory could be used to predict the flow before stall. Additionally, leading edge vortices have been shown by Ellington et al. ${ }^{8}$ to be important for insect flight. They visualized the flow about a hovering hawkmoth model and showed the formation of leading edge and tip vortices. A spiral LEV was observed along the span of the venation-like wing. They concluded that this LEV is created by the translation motion not by wing rotation. The spiral vortex, however, was not found by Birch and Dickson ${ }^{9}$ in an investigation of LEV attachment of a hovering fruit fly model, they suggested the downwash induced by the tip vortex and wake vorticity may stabilize the LEV and maintain the LEV attached during the flapping motion. Computational results of hawkmoth and fruit fly in hover by Shyy and Liu ${ }^{10}$ showed that higher Reynolds number generates more pronounced axial flow; the LEV is a helical structure and breaks down during the flapping motion. At lower Reynolds number the weaker axial flow stabilizes the LEV which is attached during the entire flapping stroke, although it breaks down during wing supination. In the present work we examine the development of aerodynamic forces and its relation to vortex development over the wing, which is relevant to biological flyers including very rapid pitch up motions relevant to aggressive maneuvers of small flyers.

2

American Institute of Aeronautics and Astronautics 


\section{Experimental Apparatus}

\section{A. Water Tunnel and Wing Configuration}

The experiments were conducted in the low-turbulence water tunnel at the University of Michigan with cross section $61 \mathrm{~cm}$ by $61 \mathrm{~cm}$. The free stream velocity ranges from $5 \mathrm{~cm} / \mathrm{s}$ to $40 \mathrm{~cm} / \mathrm{s}$. A picture of the water channel facility is shown in Fig. 1.

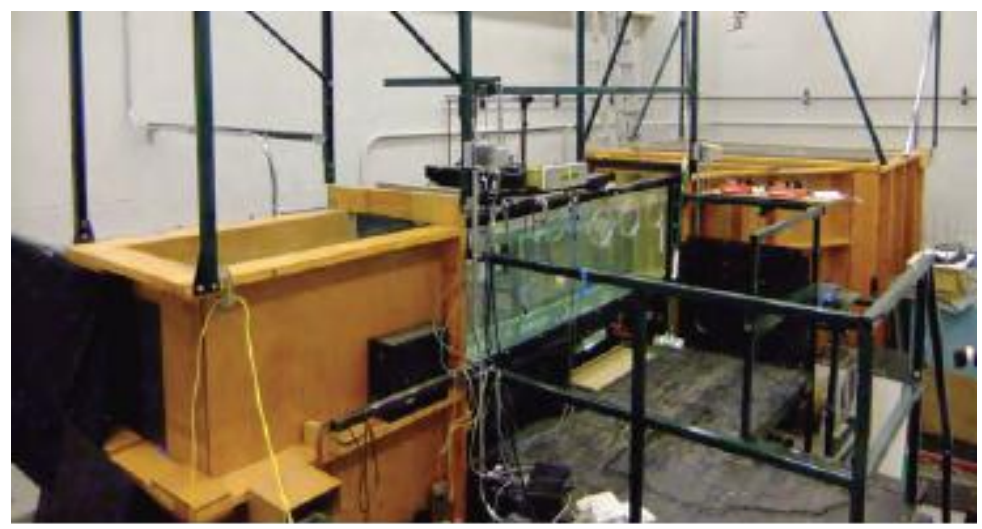

Figure 1 University of Michigan Water Channel

A finite aspect ratio wing with effective aspect ratio 4 has been tested at three pivot axis locations as illustrated in Fig. 2. The wing model is partially submerged in the water channel with the free surface providing a plane of symmetry for the flow. The pivot axis locations include: leading edge pivot (LE) illustrated at the left panel of Fig. 2(a); mid-chord pivot (ME) illustrated at the right panel of Fig. 2(a); and trailing edge pivot (TE), which uses the same wing configuration at the LE pivot with the wing being rotated 180 degrees to position the axis of rotation at the downstream edge. Fig. 2(b) illustrates the wing installation in side view. Note that the sensor's axis of symmetry is always coincident with the pivot axis, which facilitates data analysis. In all cases the force sensor is oriented to measure forces in the chord direction (x-axis), normal to the wing surface (y-axis) and in the span direction (z-axis). For the LE and ME pivot locations the measured positive $\mathrm{x}$-direction is toward the leading edge and the measured positive y direction is in the direction of positive lift force. For the TE pivot because of the 180-degree rotation, positive directions of the $\mathrm{x}$ - and $\mathrm{y}$-axes are in the opposite direction and must be converted to the normal force (denoted as N) and axial force (denoted as A) components shown in Fig. 2(b) by a simple sign change. The force sensor is a Nano 43 Force/Torque sensor manufactured by ATI Industrial Automation. The sensor's maximum calibrated load is $18 \mathrm{~N}$ and the resolution is $1 / 256 \mathrm{~N}$, the same values are in all three axis directions. The force sensor is attached to a rotary table Velmex (B4836TS) controlled by a stepper motor as described below. Both the force sensor and the rotary table are located above the water surface. The wing is a rectangular flat plate with chord length of 2" and 2 chords immersed in water. The thickness is 0.125 " (6.25\% of chord) and all edges are rounded. It is made of plexiglass sheet, mounted vertically at the center of the water channel. The wing is attached to the tool side of the sensor with an aluminum adapter designed to minimize the mass of the system. For the present experiments the total mass attached to the sensor is 41.1 grams, including the wing, the adapter, and the mounting screws. Because of the small mass, inertia forces and static weight of the model are very small which makes static calibration unnecessary. The forces measured by the sensor $\left(\mathrm{F}_{\mathrm{x}}\right.$ and $\left.\mathrm{F}_{\mathrm{y}}\right)$ are first converted to the axial and normal forces (A, N) and then to the laboratory frame of reference components using Eq. (1)-(4) for lift (L) and drag (D) respectively, and their corresponding force coefficients.

$$
\begin{gathered}
L=-A \sin \alpha+N \cos \alpha \\
D=A \cos \alpha+N \sin \alpha \\
C_{L}=L /\left(\rho U_{\infty}^{2} S / 2\right) \\
C_{D}=D /\left(\rho U_{\infty}^{2} S / 2\right)
\end{gathered}
$$




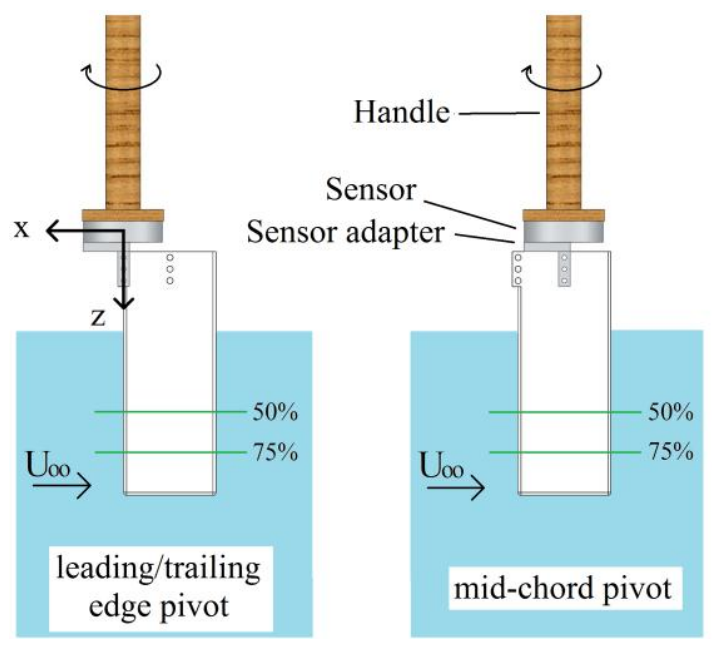

a. Top view

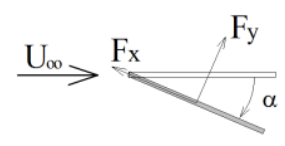

transducer frame of reference

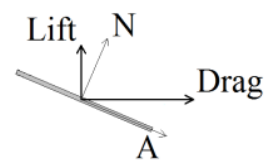

laboratory frame of reference

Figure 2. Schematic of the wing mounting in the water channel showing the relevant axes for force measurements in (a) top view (b) side view

\section{B. Wing Kinematics}

The wing motion is a linear pitch ramp from $0^{\circ}$ to $45^{\circ}$ with smoothing at the beginning and the end of rotation. For all measurements the motion is repeated 60 times to compute stable mean values with relatively small uncertainty using phase averages. Actual implementation involves a pitch ramp-hold-return motion similar to the kinematic used by $\mathrm{Yu}$ et $\mathrm{al}^{4}$. The wing motion is divided into five phases, which are the start phase, the pitch ramp phase, the hold phase, the pitch return phase, and the relaxation phase. The time duration of each phase is defined in Fig. 3. For these experiments the pitch ramp phase duration and the pitch return phase duration are the same. The duration of all the motion phases are given in Eq. (5)-(8).

$$
\begin{gathered}
\Delta T_{s}=s t_{c} \\
\Delta T_{p}=t_{p}=\alpha_{m} / \dot{\alpha}_{m} \\
\Delta T_{h}=h t_{c} \\
\Delta T_{e}=e t_{c}
\end{gathered}
$$

where $t_{c}$ is the convective time $\left(c / U_{\infty}\right), s$ is the start parameter, $h$ is the hold parameter and $e$ is the relaxation parameter. In all the cases reported here the start duration is one convective time $(s=1)$. The hold duration is at least 68 convective times $(h>68)$ to ensure the flow reached a steady condition at a pitch angle of $45^{\circ}$. The relaxation duration is about 30 convective times $(e=30)$ to return the flow to the undisturbed initial condition.

To minimize model vibration the motion was smoothed at the corners $\left(t_{1}\right.$ through $\left.t_{4}\right)$ using a modified Eldredge's function (Eldredge et $\mathrm{l}^{11}$ ) given in Eq. (9).

$$
\alpha(t)=(1 / 2 A) \ln \left\{\left[\cosh \left(A \dot{\alpha}_{m}\left(t-t_{1}\right)\right) \cosh \left(A \dot{\alpha}_{m}\left(t-t_{4}\right)\right)\right] /\left[\cosh \left(A \dot{\alpha}_{m}\left(t-t_{2}\right)\right) \cosh \left(A \dot{\alpha}_{m}\left(t-t_{3}\right)\right)\right]\right\}
$$

where $A$ is analogous to $a / 2 k$, defined in Eldredge's function ( $a$ is the free parameter and $k$ is the reduced frequency). Motion accelerations are then confined to time zones $t s 1-t s 2, t s 3-t s 4$, $t s 5-t s 6$, and $t s 7-t s 8$ as illustrated in Fig. 3.

The points used to implement the wing motion are shown as circles at the right of Fig. 3, a time step of 0.02s is used for all cases, and at least 10 points are used to capture the motion acceleration. The wing motion is 
implemented using a Velmex Rotary Table B4836TS with stepper motor model RK266-03A-P1, which provides a resolution of 40 steps/degree and a maximum pitch rate of $100 \mathrm{deg} / \mathrm{s}$.
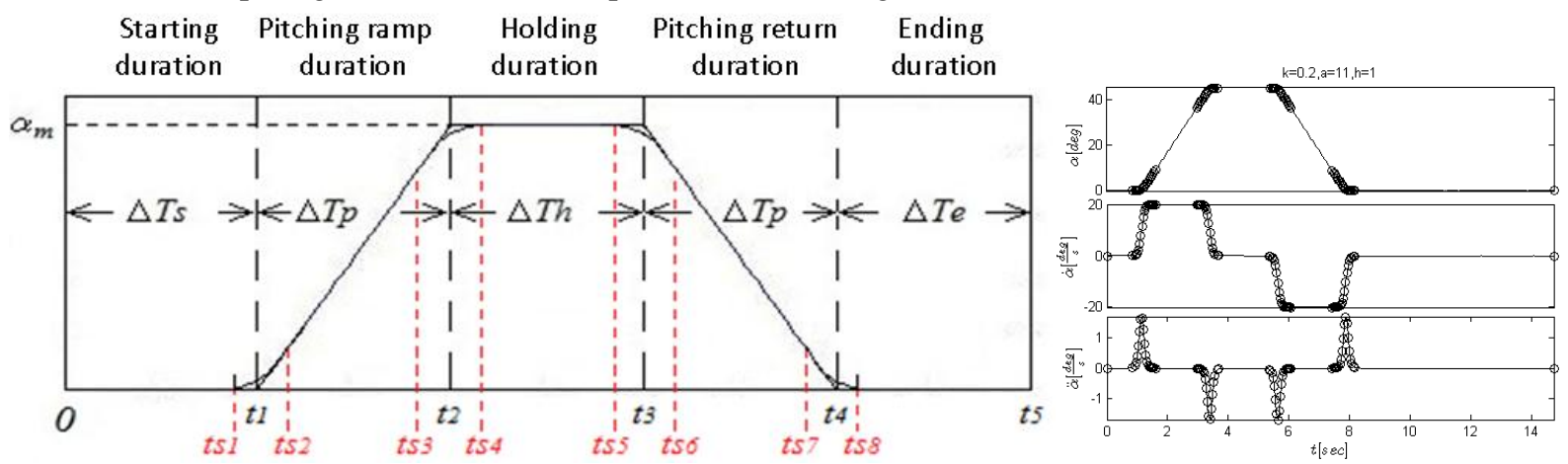

Figure 3. Schematics of wing kinematics: a linear pitch ramp-hold-return motion (left) notations and (right) an implementation. Times ts 1 to ts 8 define the smoothing transitions

\section{Test Cases for Direct Force Measurement and Data Processing}

For fixed wing geometry (i.e. aspect ratio 4 flat plate wing), maximum pitch angle $\left(45^{\circ}\right)$, pivot axis and a linear variation of pitch angle with time, there are two independent parameters that define the flow: the free stream velocity and the pitch rate. Non-dimensionalizing both parameters with relevant combinations of wing chord and fluid viscosity, the parameter space shown in Fig. 4 is obtained. The abscissa of Fig. 4 is Reynolds number $\left(U_{\infty} c / v\right)$ and the ordinate is Stokes number $\left(\dot{\alpha}_{m} c^{2} / v\right)$, which in this context is the ratio of viscous diffusion time to pitch rate characteristic time. Shown in the figure are the combinations of free stream velocity and pitch rate considered in this

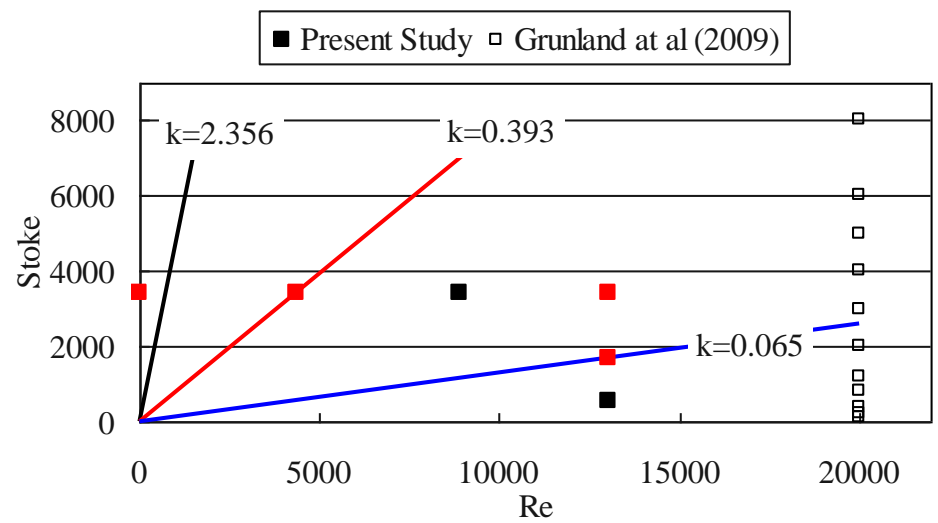

Figure 4. Test cases in Stokes number versus Reynolds number parameter space. study (square solid symbols) as well as the cases considered by Granlund $e t a l^{1}$ (square open symbols). The more important parameter for the flow dynamics is the reduced frequency which gives the ratio of flow advection time to pitch rate characteristic time. In Fig. 4 constant reduced frequency are straight lines through the origin with increased slope as reduced frequency is increased. In the present tests reduced frequency as high as 0.39 are considered which corresponds to pitch time equal to convective time for $\alpha_{\mathrm{m}}=45$ degrees. Also cases with $\mathrm{k} \rightarrow \infty$ (zero free stream velocity) corresponding to the vertical axis in Fig. 4 are of interest to examine questions of non-circulatory force and linear superposition in flow topology and force development. In the experiments all cases are repeated 60 times with sampling rate of $5000 \mathrm{~Hz}$ and ensemble averages are reported.

Conditions used to generate the three pitch rates are given in Table 1, which are $76.4 \mathrm{deg} / \mathrm{s}, 37.5 \mathrm{deg} / \mathrm{s}$, and $12.6 \mathrm{deg} / \mathrm{s}$. Free stream velocities of $8.6 \mathrm{~cm} / \mathrm{s}(R e=4.4 \mathrm{k}), 17.5 \mathrm{~cm} / \mathrm{s}(R e=8.9 \mathrm{k})$, and $25.6 \mathrm{~cm} / \mathrm{s}(R e=13 \mathrm{k})$ give reduced frequencies of interest. For constant Stokes number of 3,400 (corresponding to $76.4 \mathrm{deg} / \mathrm{s}$ ), reduced frequencies from left to right in Fig. 4 are 0.394, 0.193, and 0.132, respectively. For constant Reynolds number of $13 \mathrm{k}$, reduced frequencies from top to bottom are $0.132,0.065$ and 0.022 , respectively. The maximum angle of attack in all cases is 45 degrees. Also given in the last column of Table 1 are the pitch angles at the end of the initial acceleration as fraction of the maximum pitch angle, which is defined as the time when the rotational acceleration is $1 \%$ of the maximum acceleration. 
Table 1 Conditions to generate kinematics with three pitch rates

\begin{tabular}{cccccc}
\hline $\begin{array}{c}\dot{\alpha}_{m} \operatorname{deg} / \\
\mathrm{s}\end{array}$ & $\mathrm{A}$ & $\begin{array}{c}\mathrm{c} \\
\text { inch }\end{array}$ & $\begin{array}{c}\alpha_{m} \\
\operatorname{deg}\end{array}$ & $\begin{array}{l}\ddot{\alpha}_{t_{1}+\Delta t} \\
\ddot{\alpha}_{m}\end{array}$ & $\alpha_{t_{1}+\Delta t} / \alpha_{m}$ \\
\hline \hline $\mathbf{7 6 . 4 4 2}$ & 27.50 & & & & $13.859 \%$ \\
\hline $\mathbf{3 7 . 5 0 8}$ & 27.50 & 2 & 45 & $1 \%$ & $\frac{13.859 \%}{4.643 \%}$ \\
\hline $\mathbf{1 2 . 5 6 5}$ & 82.09 & & & & $4.643 \%$ \\
\hline
\end{tabular}

Data processing of force sensor signals includes application of a low-pass filter and a tare procedure. The lowpass filter is used to remove high frequency noise associated with structural vibrations and electronic/sensor noise. The tare procedure is used to isolate the hydrodynamic force from model inertial and model weight contributions to the measured force.

A zero-phase $1^{\text {st }}$-order 2-path Butterworth filter was used to avoid phase distortion, to reduce spurious oscillations introduced by the filter, and to provide sufficient attenuation of noise. The cutoff frequency was determined using power spectrum analysis of the motion acceleration. For pitch rates of $76.4 \mathrm{deg} / \mathrm{s}, 37.5 \mathrm{deg} / \mathrm{s}$, and $12.6 \mathrm{deg} / \mathrm{s}$, the cutoff frequencies were $8.7 \mathrm{~Hz}, 4.3 \mathrm{~Hz}$, and $4.15 \mathrm{~Hz}$, respectively. For these cutoff frequencies $90 \%$ of the frequency content of the motion acceleration is retained and the corresponding attenuation at the frequency of maximum energy content is $-5.24 \mathrm{~dB},-5.21 \mathrm{~dB}$, and $-5.40 \mathrm{~dB}$, respectively. It should be noted that the noise introduced by the rotary stage stepper motor is in the range of $100 \mathrm{~Hz}$ to $600 \mathrm{~Hz}$ and the wing vibration fundamental frequencies in air and in still water are about $50 \mathrm{~Hz}$ and $15 \mathrm{~Hz}$, respectively, significantly higher than the filter cutoff frequency.

Tare procedures are generally necessary to remove inertia and weight contributions to the measured force due to the wing acceleration and position, which include static tares and dynamic tares. The static tares are measurements in air and still water at fix angles from 0 degrees to the maximum angle of attack of interest, i.e. 45 degrees in the present study. It was found that the present wing configuration yields negligible static tares because of the very small mass of the wing and mounting hardware. The dynamic tares are measurements in air with the same kinematics as in the flow experiments. In the tare measurements the same filter cutoff frequency as in the flow experiments were used.

\section{Test Cases for 2D PIV and Data Processing}

The cases for PIV tests are shown as red square solid symbols in Fig. 4. Two pitch rates are considered, 76.4 $\mathrm{deg} / \mathrm{s}$ and $37.5 \mathrm{deg} / \mathrm{s}$. For pitch rate $76.4 \mathrm{deg} / \mathrm{s}$ experiments were conducted at Reynolds numbers $4.4 \mathrm{k}$ and $13 \mathrm{k}$, as well as in still water. For pitch rate $37.5 \mathrm{deg} / \mathrm{s}$ only Reynolds number of $13 \mathrm{k}$ was investigated. The laser sheet was positioned normal to the pivot axis (horizontally in the lab frame) at $50 \%$ and $75 \%$. These locations are shown as green horizontal lines in Fig. 4(a). The wing used for PIV was the same as for the direct force measurements. The same pivot axis locations were used in the PIV measurements. The images were taken during the pitch ramp phase at times shown as vertical lines in Fig. 5 which correspond to 3deg, 13deg, $22.5 \mathrm{deg}, 32 \mathrm{deg}$, and $42 \mathrm{deg}$ for both pitch rates. The blue and red vertical lines in Fig. 5 are the times where the motion stops at 45 deg for the pitch rate of $37.5 \mathrm{deg} / \mathrm{s}$ and $76.4 \mathrm{deg} / \mathrm{s}$, respectively.

The PIV system includes a double-pulsed Nd-YAG laser (Spectra Physics PIV 300), light sheet optics, a dual frame digital camera (Cooke Corp. PCO.4000), a computer image acquisition system and control electronics. The thickness of the laser sheet is about $0.8 \mathrm{~mm}$. The water channel was seeded using Titanium Dioxide particles (Sigma-Aldrich) with diameter less than $5 \mu \mathrm{m}$. The PIV camera was installed underneath the water channel test section and is equipped with a Nikon 105-mm Micro-Nikkor lens to produce a magnification of 16.1 pixels $/ \mathrm{mm}$. Therefore, the PCO.4000 camera frame size of 4008 by 2672 pixels gives a field of view of $248 \mathrm{~mm}$ by $165 \mathrm{~mm}$. The exposure time was selected to produce a particle displacement of 2 pixels in order to minimize particle loss due to spanwise flow. To produce a uniform distribution of particles a small amount $(1 \mathrm{cc})$ of dispersant (DARVAN C$\mathrm{N}$, Vanderbilt) was added to the particles and a Sonicator was used to produce a uniform distribution of particles.

An in-house developed MATALB-based PIV software was employed to analyze the PIV images. The particle displacement is determined using cross-correlation analysis between displaced interrogation windows. Particle displacement measurements are validated using two criteria: the correlation peak value must be large compared to the noise level in the cross-correlation function, and a region of interest criterion. A two-pass procedure is used to increase the spatial resolution of the measurement. The first pass uses a 64 by 64 pixels non-displaced interrogation 
window, and the second high-resolution pass uses a 32 by 32 pixels displaced interrogation window, the displacement is measured in the first pass. The flow velocity is measured in a uniform grid with 16 pixel spacing. A median filter is used to remove outliers and to interpolate points where the validation criteria failed. The time between exposures was selected to minimize the number of failed validation points due to spanwise flow. Based on these PIV parameters the spatial resolution of the PIV measurements is approximately $1 \mathrm{~mm}$. A total of 60 PIV image pairs were recorded at each flow condition and wing angle. Results presented here are phase averages over the entire 60 image samples.

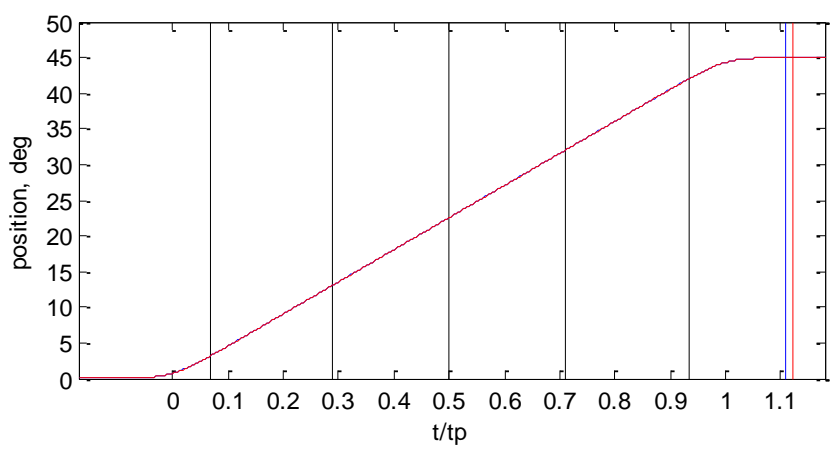

Figure 5. Locations of the snapshots' for PIV measurement during the pitch ramp phase.

\section{Results and Discussion}

In this section the force data are examined in terms of convective time, pitching time, and angle of attack. The PIV data are presented to document the relation between flow topology and aerodynamic force development.

\section{A. Axial Force and Normal Force Evolution as Functions of Convective Time}

Figures 6, 7 and 8 show axial and normal forces as a function of convective time (i.e. physical time with origin at $t_{l}$ normalized by the advection time $t_{c}=c / U$ ) for LE, ME and TE pivot locations respectively. The solid curves represent cases with pitch rate $76.4 \mathrm{deg} / \mathrm{s}$; the corresponding wing position is shown as a dotted red curve. The blue, green and red solid lines correspond to Reynolds numbers $4.4 \mathrm{k}, 8.9 \mathrm{k}$, and $13 \mathrm{k}$, respectively. The blue and black dashed lines correspond to pitch rate $37.5 \mathrm{deg} / \mathrm{s}$ and $12.6 \mathrm{deg} / \mathrm{s}$, respectively, and Reynolds number of $13 \mathrm{k}$; the corresponding wing angle of attack lines are plotted as blue and black dotted curves, respectively. Error bars are plotted after the wing has reached 45 degree angle of attack and are representative of the $95 \%$ confidence intervals of these measurements. The $95 \%$ confidence interval is of the order of $10 \mathrm{mN}$ or less for both axial and normal force. For the axial force this error is small but significant compared to the measured force, for the normal force this value is very small compared to the measured normal force. As expected error bars are larger at higher free stream velocity and for cases with leading and trailing edge pivot axis. Note also that the forces for the low Reynolds number cases are very small due to small dynamic pressure.

The data in Fig. 6 - 8 show that the normal force increases rapidly during the pitch-up motion, followed by a decrease until a constant steady state value is reached. The decrease in normal force shows some oscillatory behavior at early times, which is in phase with the axial force oscillation, with an increase in normal force occurring at the same phase as a more negative axial force (i.e. increased axial force towards the leading edge). This is consistent with an increase of leading edge suction associated with a lower pressure on the leeward side of the plate. The period of these oscillations is consistent with a transient vortex shedding process. The oscillations disappear before steady state is reached, which suggests that there is no vortex shedding at steady state conditions. Close examinations of individual realization do not show oscillatory behavior at steady state conditions, which supports this conclusion. The time when the maximum normal force occurs depends on pivot axis location and reduced frequency. These differences will be discussed below in terms of force coefficients. Fig. $6-8$ also show the measured axial force. The magnitude of the axial force is two orders of magnitude smaller than the magnitude of the normal force. Axial force reaches a steady state value at approximately the same convective time as the normal force $\left(t / t_{c}>40\right)$. The axial force at steady state is negative which implies forward force and significant leading edge suction. The magnitude of the leading edge suction force is very low compared to potential flow theory estimates, as expected for separated flow at the leading edge. It is not clear at this point how this suction force depends on the geometry of the leading edge (e.g. a sharper leading edge may promote leading edge suction). 

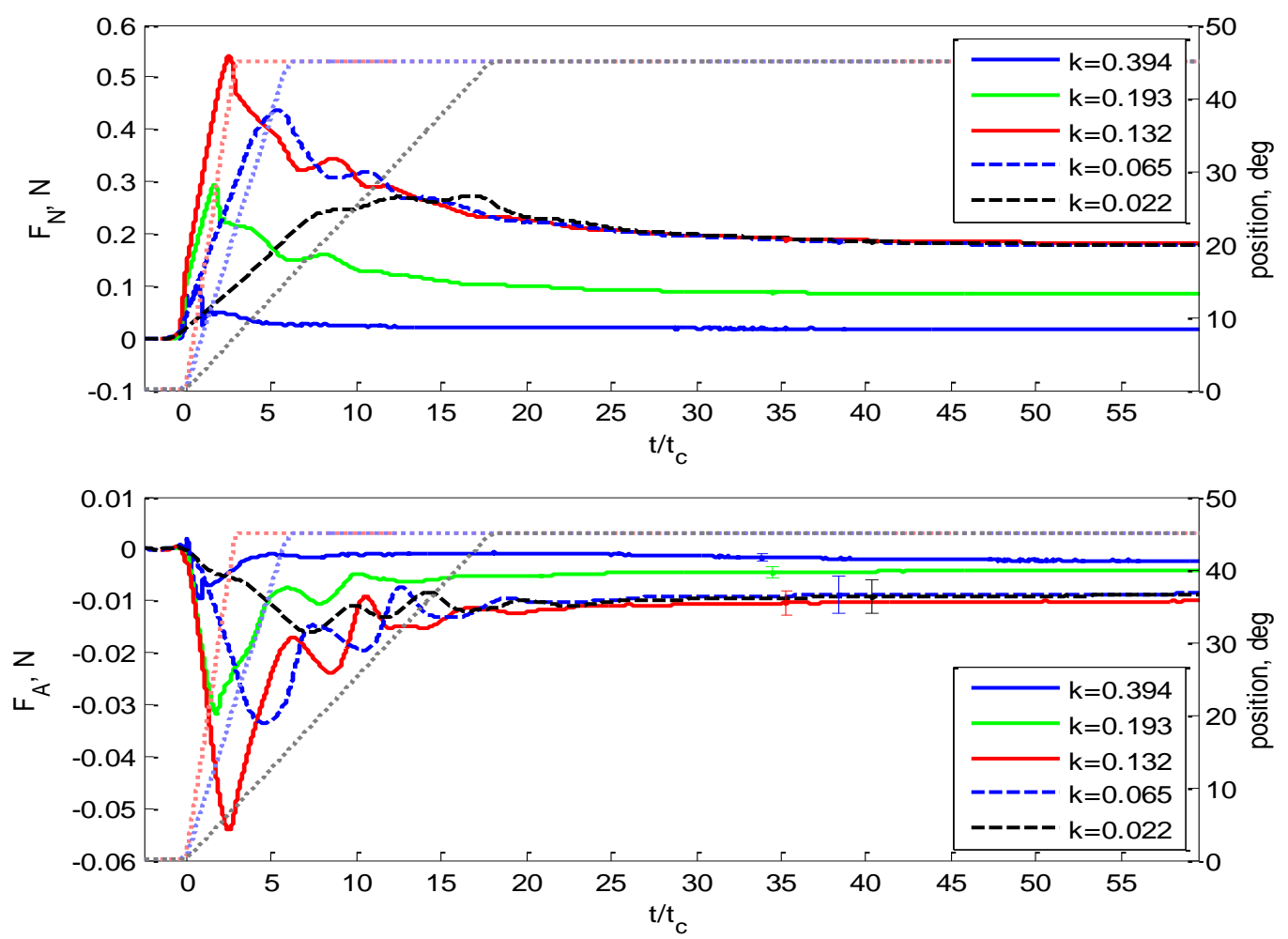

Figure 6. Force evolution as a function of normalized convective time at leading edge pivot (top) normal force (bottom) axial force
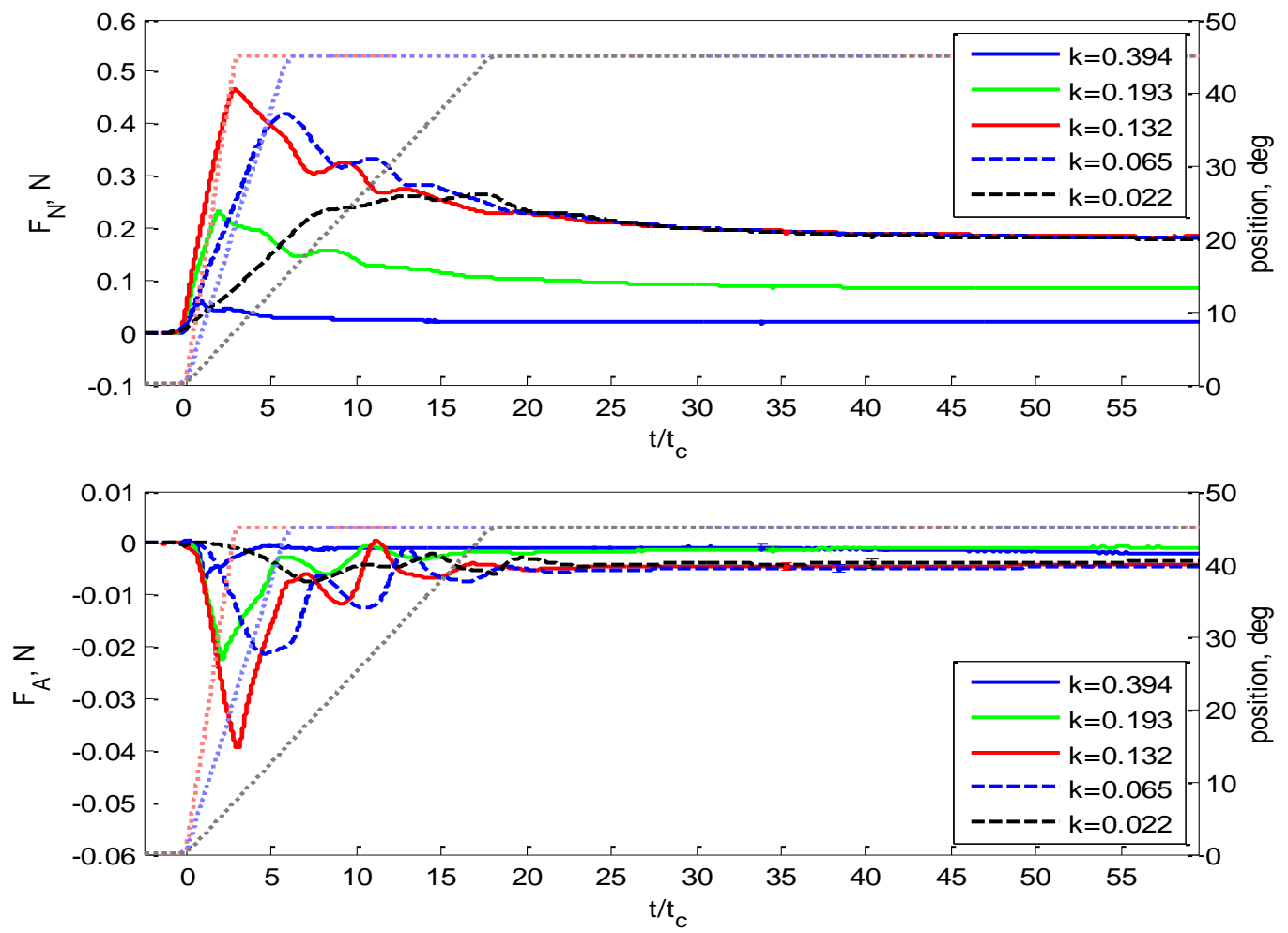

Figure 7. Force evolution as a function of normalized convective time at mid-chord pivot (top) normal force (bottom) axial force 

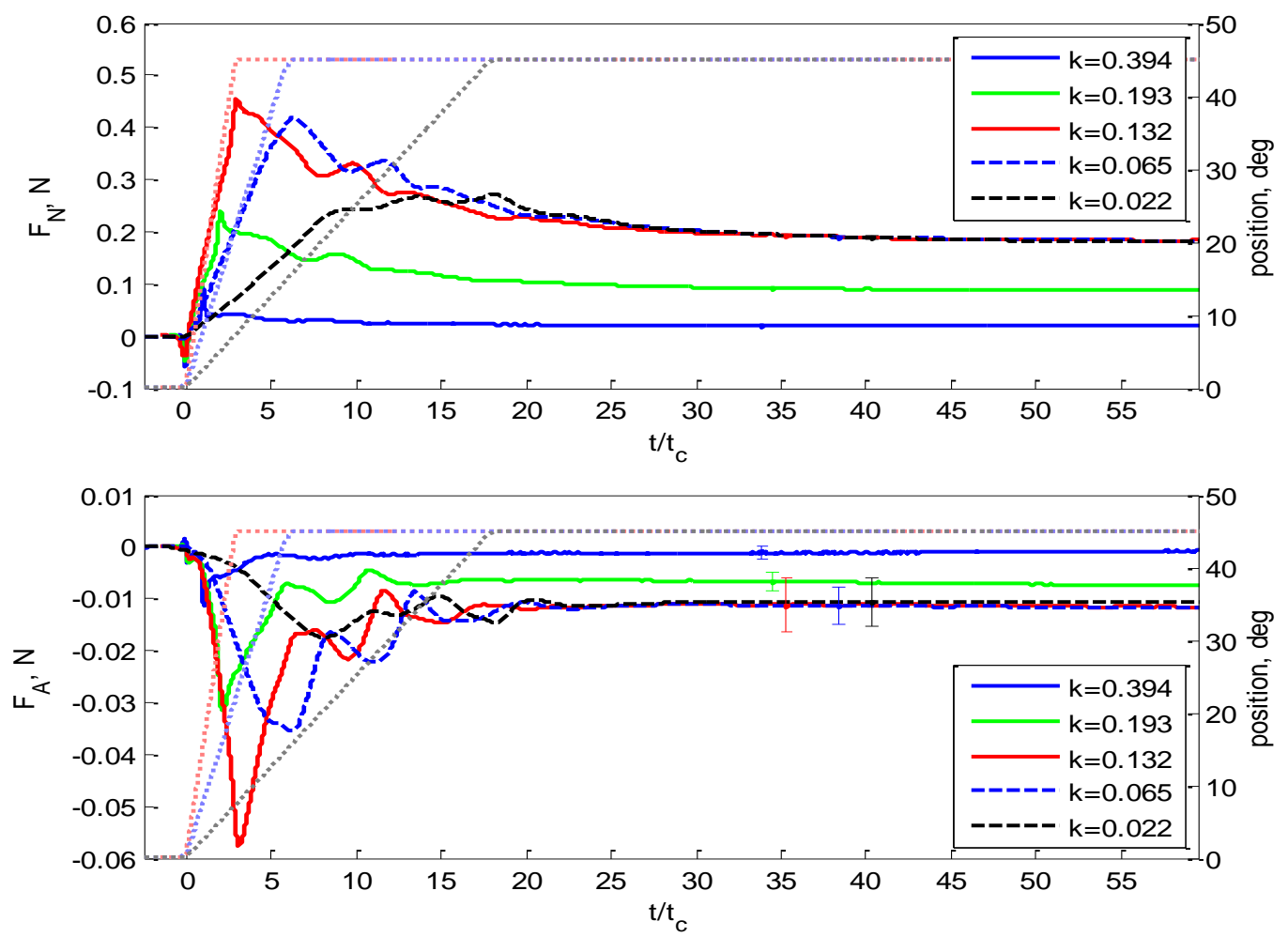

Figure 8. Force evolution as a function of normalized convective time at trailing edge pivot (top) normal force (bottom) axial force

Figure 9 illustrates the effect of pivot axis on normal force coefficients for $R e=13 \mathrm{k}$. The solid lines are for leading edge pivot, and the dashed and dotted curves are for the mid-chord and trailing edge pivot, respectively. The red, blue, and black lines represent the pitch rate of $76.4 \mathrm{deg} / \mathrm{s}, 37.5 \mathrm{deg} / \mathrm{s}$, and $12.6 \mathrm{deg} / \mathrm{s}$, respectively. The corresponding reduced frequencies are 0.132, 0.065, and 0.022, respectively. All cases show that normal force coefficients converge to the same value of approximately 1.1 after at least 30 convective times. Leading edge pivot axis produces larger normal force coefficients compared to mid-chord pivot axis, which in turn produces larger normal force coefficients compared to trailing edge pivot axis. At low reduced frequency $k=0.022$ the normal force coefficient increases rapidly until the wing reaches an angle of about 20 degrees at 8 convective times and remains slightly above this value for the remaining pitch up time. At this lower reduced frequency pivot axis has very small effect on normal force coefficient. At higher reduced frequency the effect of pivot axis location is more pronounced. At higher reduced frequency the maximum normal force coefficient occurs at approximately the maximum angle of attack and depends on the pivot axis location. Figure 9 also shows the oscillatory behavior noted earlier for convective times in the range $4 \sim 16$ which is attributed to transient vortex shedding. 


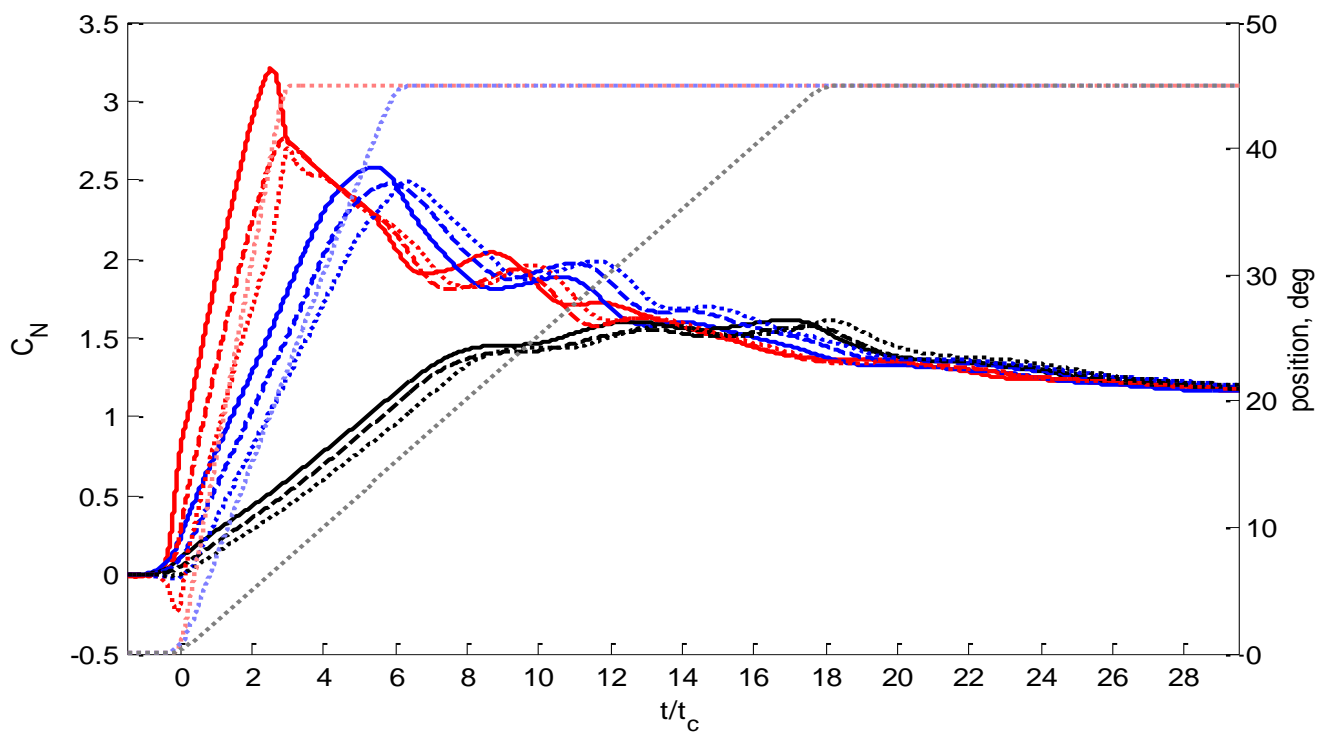

Figure 9. A comparison of normal force evolution as a function of normalized convective time at selected pivot locations. The solid, dashed, and dotted curves represent the leading edge pivot, the mid-chord pivot, and the trailing edge pivot, respectively. The red, blue, and black curves denote the cases for $k=0.132, k=0.065$, and $k=0.022$, respectively.

\section{B. Analysis of Non-Circulatory Effects}

Figure 10,11 and 12 show the normal force on the wing as a function of pitch time normalized by the pitch ramp duration; the pitch ramp phase $\left(t_{1}-t_{2}\right)$ is labeled from 0 to 1 . In this time scale the non-circulatory (apparent mass) effects during the acceleration at the start of rotation and the deceleration at the end of rotation occur at the same values as denoted by non-dimensional time 0 and 1 , respectively. Between these two values the wing pitches at constant rate. Also shown in these figures is the force measured at zero free stream velocity at pitch rate $76.4 \mathrm{deg} / \mathrm{s}$, denoted as solid black curves. The blue, green, and red curves are corresponding to Reynolds number $4.4 \mathrm{k}, 8.9 \mathrm{k}$, and $13 \mathrm{k}$, respectively. For the pitch rates $37.5 \mathrm{deg} / \mathrm{s}$ and $12.6 \mathrm{deg} / \mathrm{s}$ at Reynolds number of $13 \mathrm{k}$, the blue and black dashed curves are used. The corresponding motion accelerations are shown in the blue and black dotted lines.

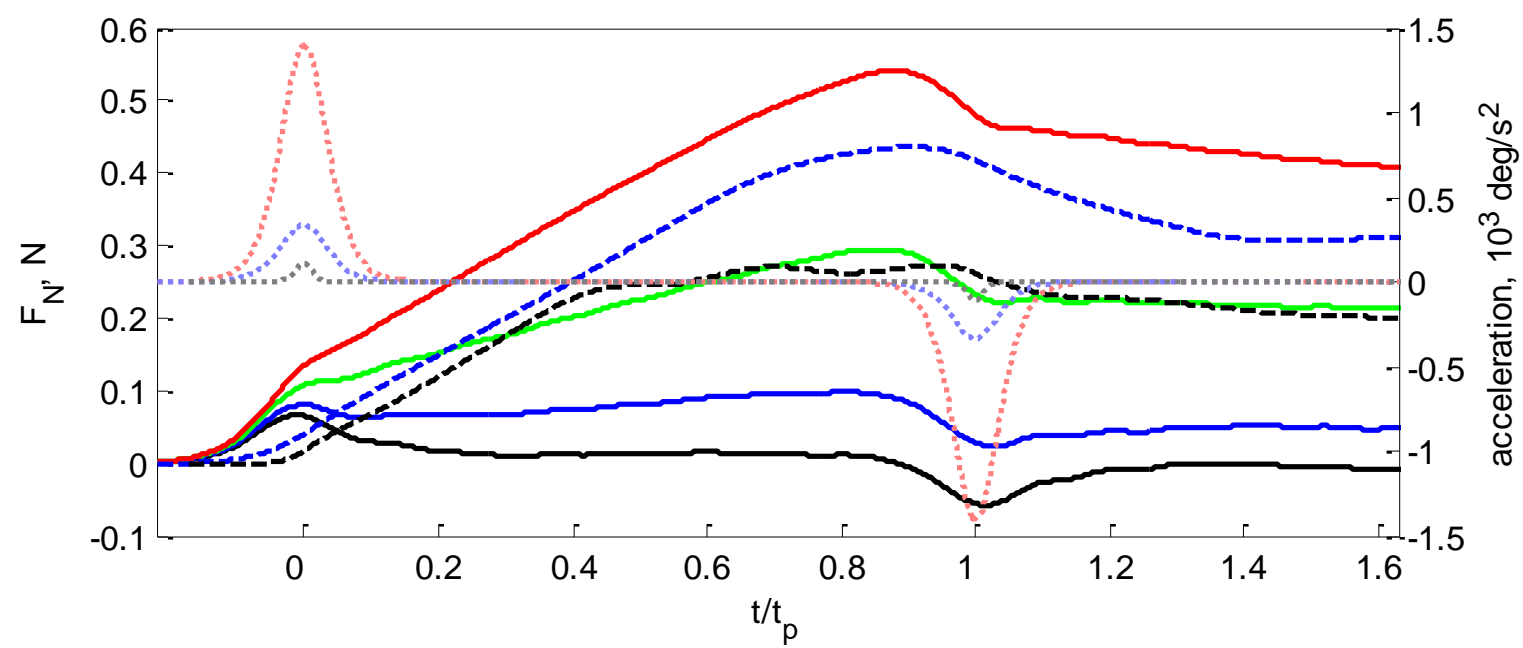

Figure 10. Normal force evolution as a function of normalized pitching time at leading edge pivot. The black solid curve is for $k=\infty$, the blue solid curve is for $k=0.394$, the green solid curve is for $k=0.193$, the red solid curve is for $k=0.132$, the blue dashed curve is for $k=0.065$, and the black dashed curve is for $k=0.022$.

Figure 10 shows the normal force for leading edge pivot axis as a function of pitching time. The main feature in this plot is the non-circulatory apparent mass spikes occurring at the start and the end of rotation for reduced frequencies above 0.065. These spikes have been documented by many researchers (Eldredge et al in 2009, 
Granlund et al in 2010, Ramesh et al in 2011 and others). The normal force spike is positive at the start of rotation and negative at the end of rotation as would be expected from simple consideration of mass loading due to rotational acceleration. The magnitude of the spikes increases with the free stream velocity at the start of rotation, which suggests that non-circulatory apparent mass effects are enhanced by circulatory rotation rate effects. The constant pitch rate part of the motion between pitching time 0 and 1 shows an increase in normal force with time (i.e. angle of attack). The slope increases as reduced frequency decreases with increasing free stream velocity. This part of the motion will be discussed in detail in the next section in terms of lift and drag coefficients as functions of angle of attack.

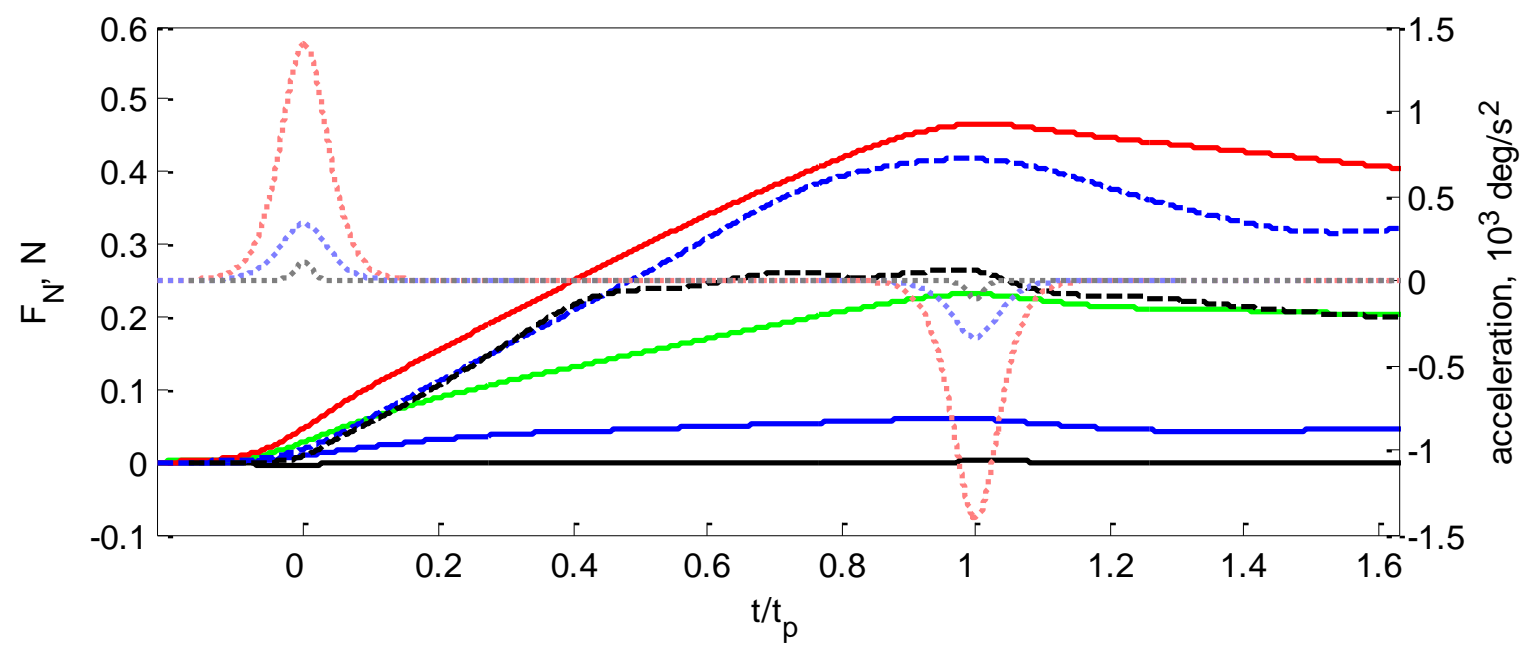

Figure 11. Normal force evolution as a function of normalized pitching time at mid-chord pivot. The black solid curve is for $k=\infty$, the blue solid curve is for $k=0.394$, the green solid curve is for $k=0.193$, the red solid curve is for $k=0.132$, the blue dashed curve is for $k=0.065$, and the black dashed curve is for $k=0.022$.

Figure 11 shows the normal force for mid-chord pivot axis as a function of pitching time. In this case there is no non-circulatory contribution to the normal force, as would be expected from symmetry of the rotation acceleration. It should be noted that non-circulatory apparent mass effects are not found at the end of rotation where circulatory effects must have introduced asymmetries between the leading edge and the trailing edge.

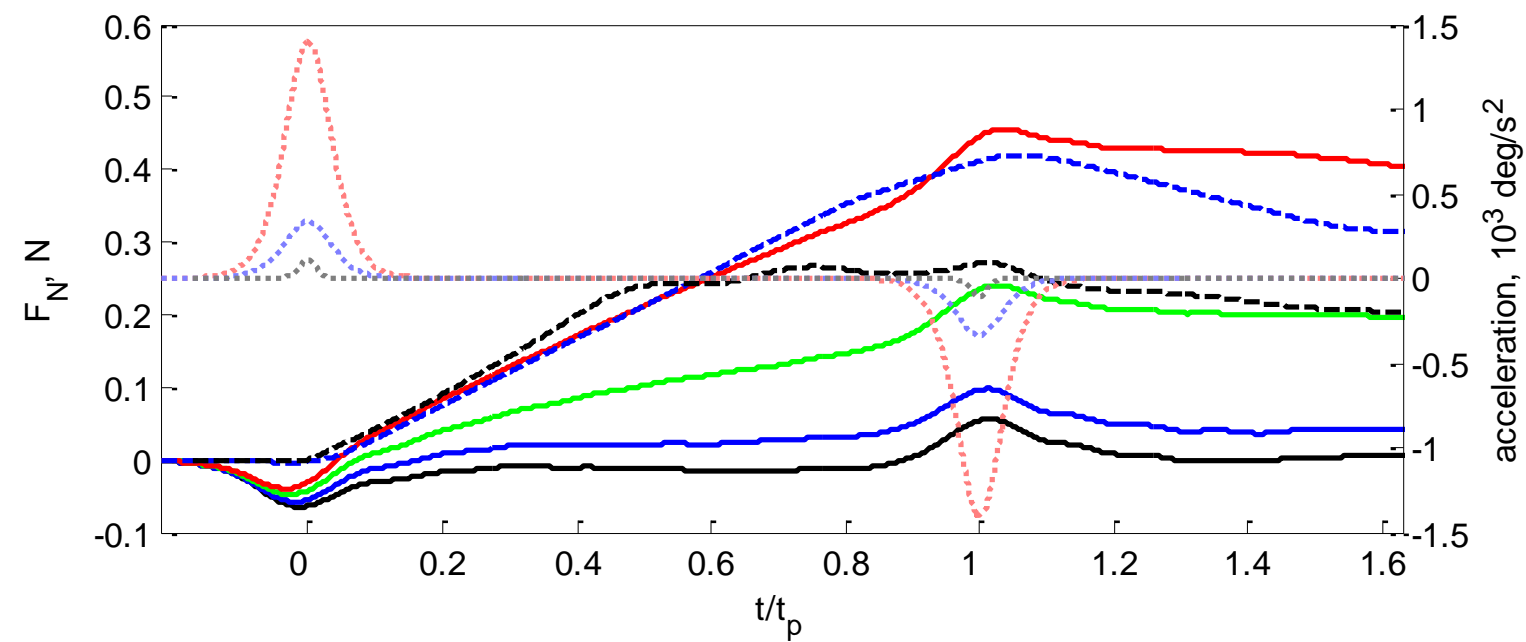

Figure 12. Normal force evolution as a function of normalized pitching time at trailing edge pivot. The black solid curve is for $k=\infty$, the blue solid curve is for $k=0.394$, the green solid curve is for $k=0.193$, the red solid curve is for $k=0.132$, the blue dashed curve is for $k=0.065$, and the black dashed curve is for $k=0.022$.

Figure 12 shows the normal force for trailing edge pivot axis as functions of pitching time. In this case noncirculatory effects are in the opposite direction compared to leading edge pivot axis. A negative normal force spike is produced at the start of rotation and a positive spike is produced at the end of rotation for $\mathrm{k}>0.065$. The magnitude of the spike decreases with the increasing free stream velocity in contrast with the results for leading edge pivot axis, which suggests that non-circulatory apparent mass effects at the start of rotation are reduced by 
circulatory rotation. However the increase in normal force during the constant pitch rate portion of the motion shows the same trend of increasing slope with reduced frequency as for leading edge pivot axis.

\section{Lift and Drag Coefficients as Functions of Angle of Attack}

Now we consider the dependence of lift and drag coefficients on angle of attack. The main motivation for this analysis is to ascertain the significance of unsteady flow effects compared to well-established steady flow results. A similar analysis was conducted by Granlund $e t a l^{1}$ for a flat plate airfoil (2D) in perching motion up to 90 degrees angle of attack of, and $\mathrm{Yu} e t \mathrm{al}^{4}$ for pitching motion of a plate airfoil at several maximum angles of attack and a reduced frequency $k=0.2$. Fig. 13, 14 and 15 show the lift and drag coefficients as a function of angle of attack for leading edge, mid-chord and trailing edge pivot axes, respectively. The solid lines are cases with pitch rate 76.4 $\mathrm{deg} / \mathrm{s}$ at Reynolds numbers of $4.4 \mathrm{k}, 8.9 \mathrm{k}$, and $13 \mathrm{k}$, the corresponding force coefficients are plotted in blue, green, and red, respectively. The blue and black dashed lines are the force coefficients for the pitch rates of $37.5 \mathrm{deg} / \mathrm{s}$ and $12.6 \mathrm{deg} / \mathrm{s}$, respectively, at Reynolds number 13k. The motion acceleration for the pitch rate of $76.4 \mathrm{deg} / \mathrm{s}, 37.5$ $\mathrm{deg} / \mathrm{s}$, and $12.6 \mathrm{deg} / \mathrm{s}$ are plotted in red, blue, and black dotted lines, respectively. Also shown in these plots are estimates of lift and drag coefficient based on lifting-line theory, which assumes steady inviscid flow. The slope of the lift coefficient vs. angle of attack curve and the induced drag coefficient are given in Eq. (10) and Eq. (11), respectively, for a wing of arbitrary planform shape.

$$
\begin{gathered}
\frac{d C_{L}}{d \alpha}=a=\frac{a_{0}}{1+\left(a_{0} / \pi A R\right)(1+\tau)} \\
C_{D, i}=\frac{C_{L}^{2}}{\pi e A R}
\end{gathered}
$$

where $e$ is a span efficiency factor $(e=1 /(1+\delta))$, the parameters $\delta$ and $\tau$ were introduced by Glauert (see Anderson ${ }^{12}$ ) to characterize departure from the optimal elliptical lift distribution. For a rectangular wing of aspect ratio $A R=4$ the values of $\delta$ and $\tau$ are small, approximately 0.04 (Anderson ${ }^{11}$ ), and can be neglected for the present discussion. The main effect for aspect ratio $A R=4$ is the slope of lift coefficient curve being factor of $2 / 3$ of the $2 \mathrm{D}$ lift coefficient slope. Lifting line theoretical results are plotted as black solid lines in Fig. 13-15 and are labeled as lifting-line theory. Finally, also shown as vertical lines are the angles of attack at which PIV data were obtained, which will be discussed in the next sections. 

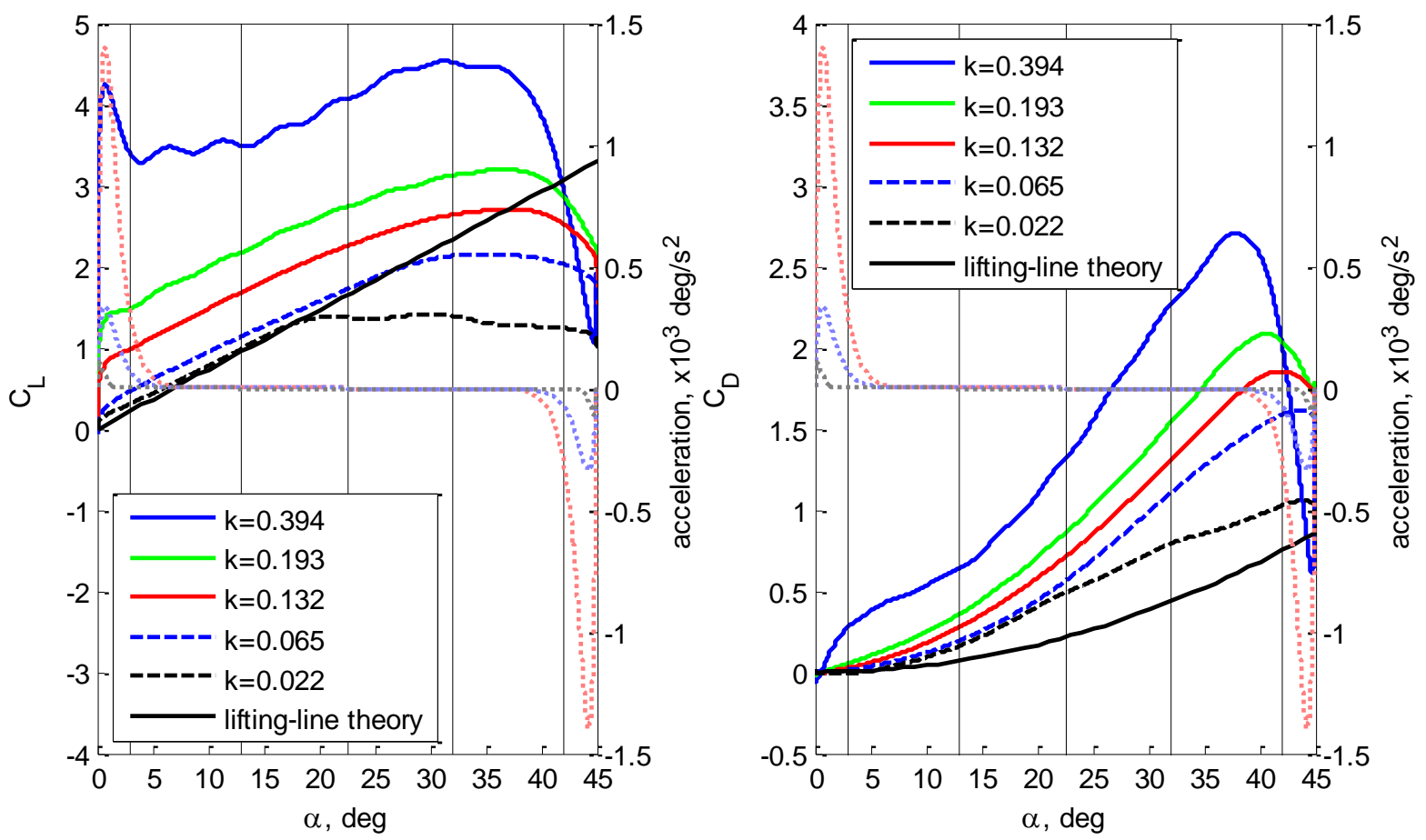

Figure 13. Force evolution as a function of angle of attack at leading edge pivot (left) lift coefficient (right) drag coefficient

Figure 13 shows the lift and drag coefficients for leading edge pivot axis. The lift coefficient results show several important features. At the lowest reduced frequency (i.e. $k=0.022$ ) the lift coefficient follows the theoretical result closely up to an angle of attack of 20 degrees, which corresponds to the steady stall angle of attack. The drag coefficients is significantly higher than the prediction by the lifting line theory, a better estimate consistent with the relatively small value of the leading edge suction is assumed that the aerodynamic force is normal to the plate, which follows the dashed line in the right panel of Fig. 13. For larger angles of attack the drag coefficient increases while the lift coefficient remains approximately constant which implies smaller L/D values. These data suggest that for $\mathrm{k}<0.022$ the flow is quasi-steady and the lifting line theory provides good estimates of the lift coefficients up to the stall angle of attack. In this regime the drag coefficient is under predicted by the lifting line theory by a factor of two. Thus the lifting line theory appears to correctly account for finite wing effects in the slope of the lift curve (i.e. a 33\% reduction of the lift coefficient according to the infinite wing), but fails to account for the significant reduction in leading edge suction which results in largest drag coefficients compared to induced drag calculations.

For $\mathrm{k}>0.022$ unsteady effects are important. There are two main effects for the lift coefficient: a non-circulatory peak during wing acceleration, and rotation rate effects within regions of constant pitch rate, the lift coefficients shift upward with the same amounts as reduced frequency increases. Similar effects were documented for 2D flow by Granlund $e t a l^{1}$. Both effects are very strong at much higher reduced frequency $k=0.394$. As noted earlier, noncirculatory effects produce a positive spike at the start of rotation, and a negative spike at the end of rotation. The shift in the lift coefficient curve due to rotation rate effect is similar to mean camber effects as would be expected from linear potential flow theory. Unsteady effects result in an increase in drag coefficient. At $k=0.394$ there is a significant increase of drag at small angle of attack during the acceleration part of the motion. 

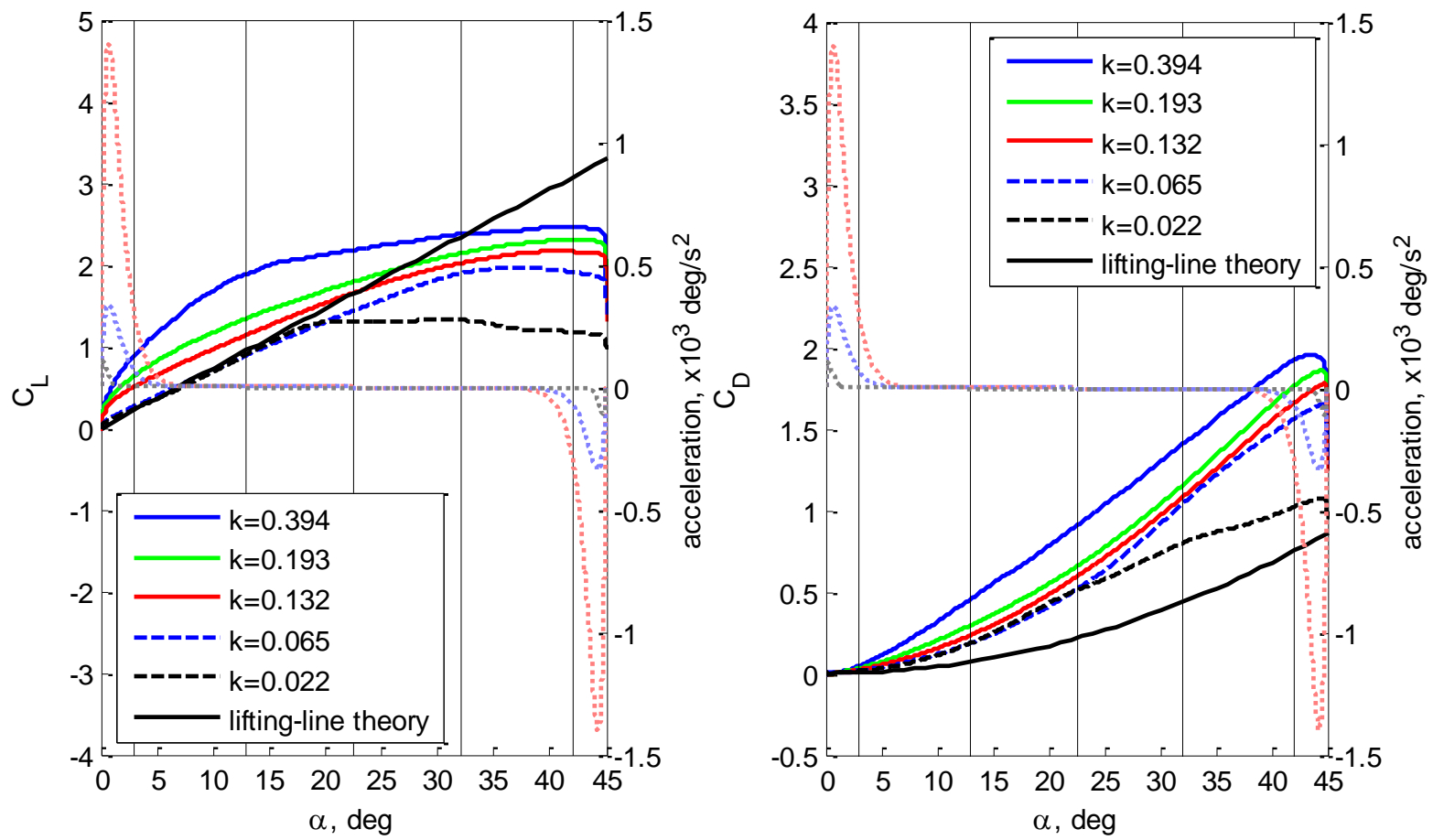

Figure 14. Force evolution as a function of angle of attack at mid-chord pivot (left) lift coefficient (right) drag coefficient

Figure 14 shows the lift and drag coefficients at mid-chord pivot axis. At the lowest reduced frequency $(k=$ $0.022)$ the lift coefficient follows the theoretical results closely up to an angle of attack of 20 degrees. The drag coefficient follows similar trends as for the leading edge pivot axis. These data confirm the conclusion that for $\mathrm{k}<$ 0.022 the flow is quasi-steady and the lifting line theory provides good estimates of the lift coefficient up to the stall angle of attack.

For values of $\mathrm{k}>0.022$ unsteady effects are important. However the trends are different compared to leading edge pivot axis. In this case non-circulatory effects are very small. Rotation rate effects between the initial and final acceleration are much stronger at $\mathrm{k}>0.065$, which increases the lift coefficient at small angles of attack. Within the constant pitch rate region, the lift coefficient curves are likely shifting upward as reduced frequency increases, but not with the same amount for leading edge pivot axis. The drag coefficient shows small change in the drag coefficient for $\mathrm{k}<0.39$. The case $k=0.394$ shows larger effects in both the lift and drag coefficients. 

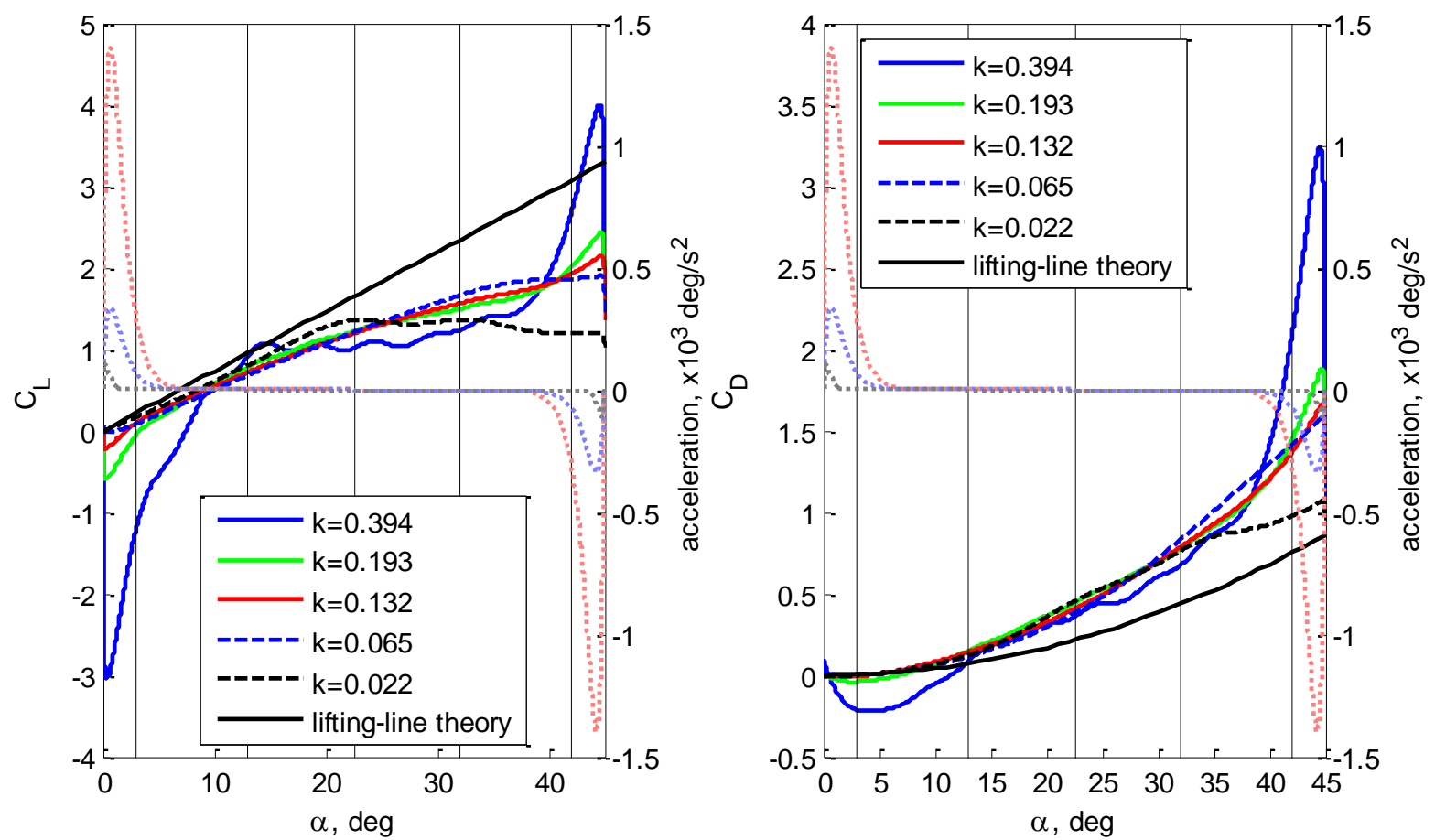

Figure 15. Force evolution as a function of angle of attack at trailing edge pivot (left) lift coefficient (right) drag coefficient.

Figure 15 shows the lift and drag coefficients for trailing edge pivot axis. At the lowest reduced frequency $(k=$ 0.022 ) the lift coefficient and drag coefficient show similar quasi steady behavior as for other pivot axes. At higher reduced frequency the lift coefficients are less than the estimation from the lifting line theory, and not very different compared to the results at $k=0.022$, except for $k=0.394$. Non-circulatory effects result in a negative lift coefficient at the beginning of rotation and a positive spike at the end of rotation. The magnitude of the spike is moderate except for the $k=0.394$ case. For $k=0.394$ the initial acceleration persist well beyond the end of the acceleration period where the lift coefficient is negative. The drag coefficient is also negative in this region.

\section{PIV Velocity Field Measurements}

Mean vorticity fields for $k=\infty, 0.394,0.132$ and 0.065 and leading edge, mid-chord and trailing edge pivot axes are compiled in Appendix A. Selected PIV images will be discussed here to illustrate the main topological features of the flow fields for the different cases and the relation of these features to the aerodynamic forces. The contour level of vorticity ranges from -0.2 to 0.2 . Also shown in these plots are $2 \mathrm{D}$ streamlines computed by assuming that the out-of-plane velocity is zero, which is clearly an incorrect assumption. However these streamlines are a useful way to identify regions of strong downwash which provides valuable insight on flow topology.

Figures 16 through 21 illustrate pivot axis effects for different flow conditions and two spanwise locations at two angles of attack 22.5 and 45 degrees. Each figure shows PIV vorticity fields for the same pivot axis and angle of attack. Also shown in these plots are results for $k=\infty$ which corresponds to zero free stream velocity. 

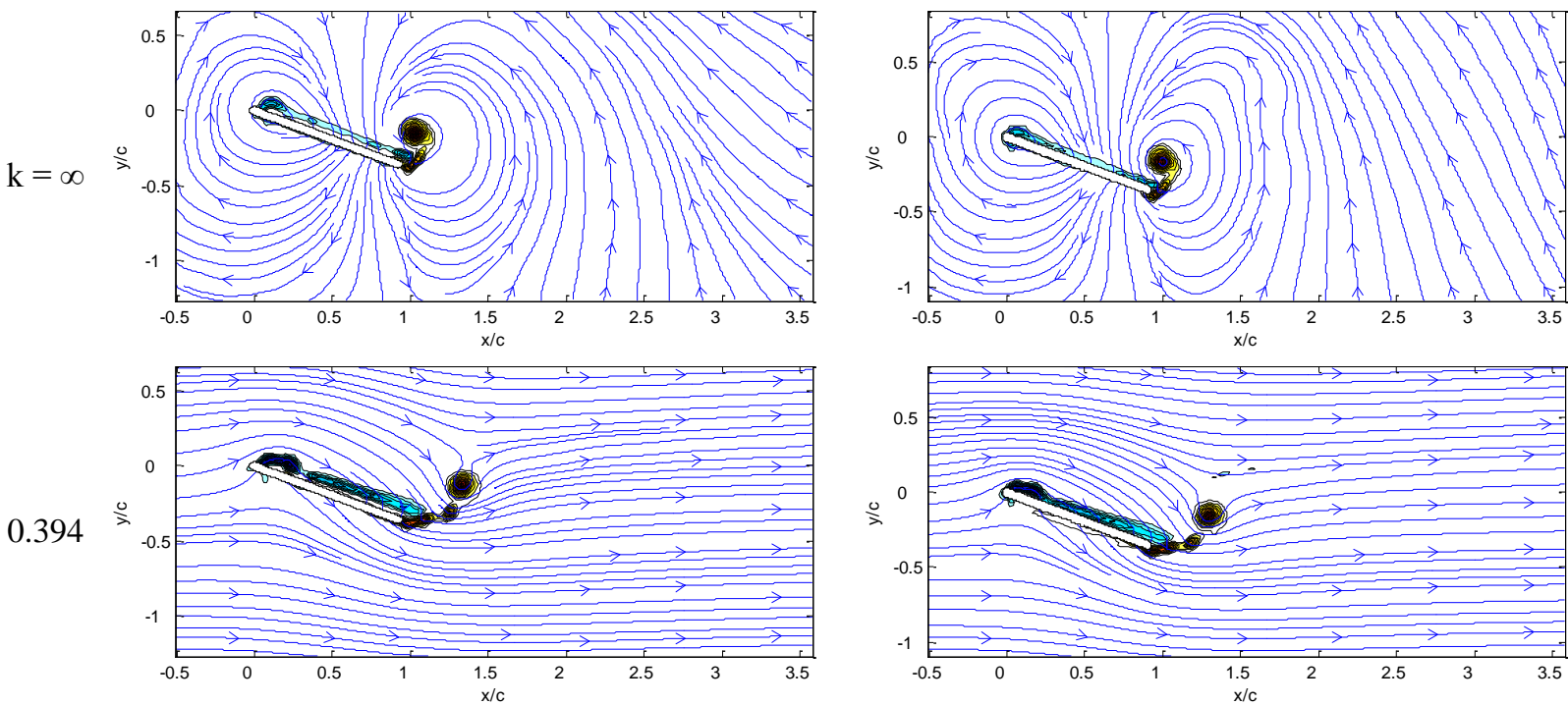

0.394
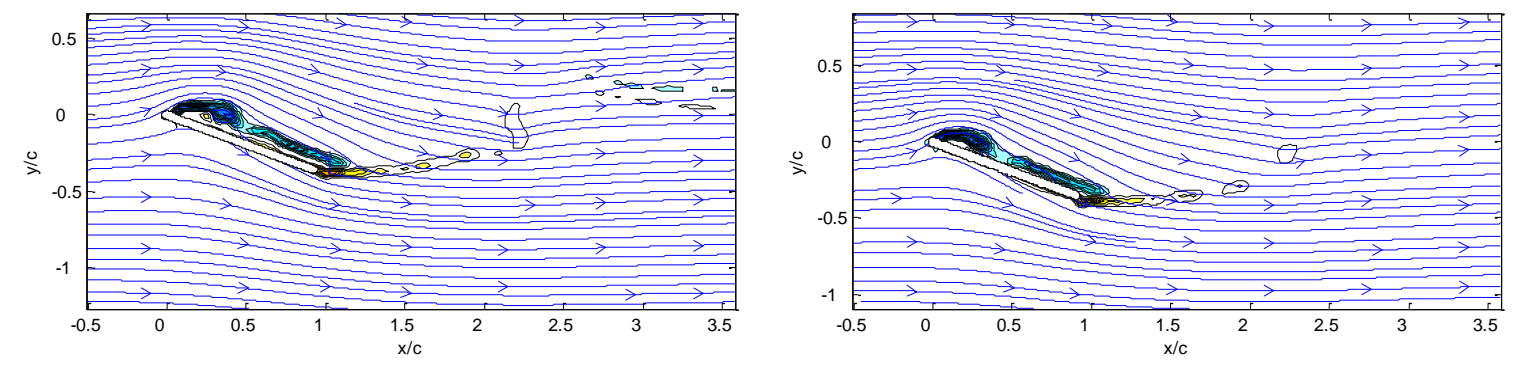

0.132
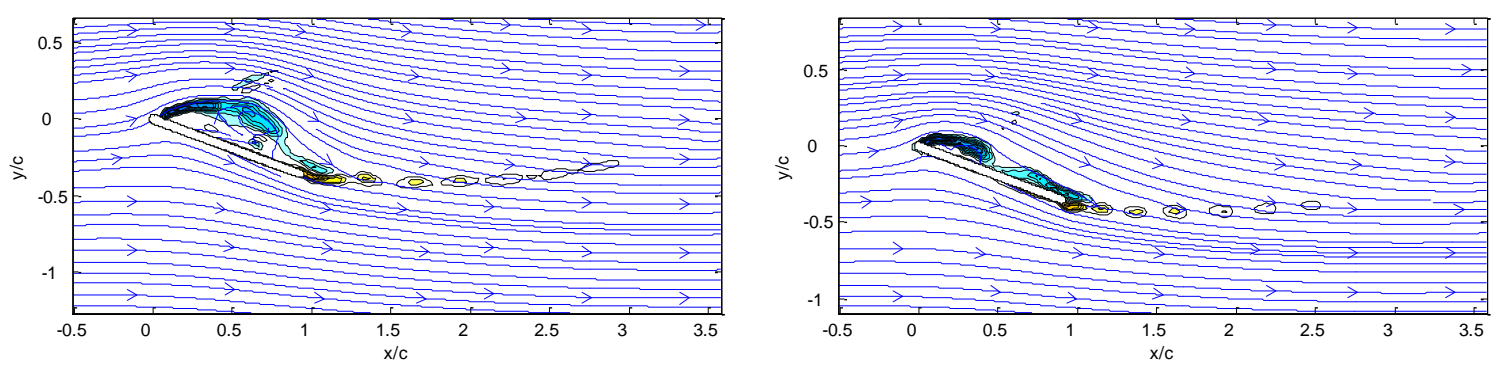

Figure 16. Vorticity fields for leading edge pivot axis at angle of attack 22.5 degrees and reduced frequency as indicated in the left column. The vorticity field at $50 \%$ span is shown on the left and the vorticity field at $75 \%$ span is shown at the right.

For pivot axis at the leading edge, Fig. 16 and 17 show the development of leading edge vortex (LEV) and trailing edge vortex (TEV) at $22.5 \mathrm{deg}$ and $45 \mathrm{deg}$, respectively. The first common features of both figures is, for the still water case $(k=\infty)$, the starting vortex (having counterclockwise vorticity) formed at trailing edge during the pitch ramp phase. Secondly, spanwise changes of flow developments are small by comparing images at different span locations, the flow is likely two-dimensional. As reduced frequency decreases to 0.39 , the spanwise variations are still small even at higher angle of attack, the flow is also likely two-dimensional.

At lower angle of attack $22.5 \mathrm{deg}$ (i.e. Fig. 16) and for high and moderate reduced frequency, LEV vortex is not observed and the flow is two-dimensional. At lowest reduced frequency $k=0.065$ the LEV develops and a separated flow region is observed at the leading edge, this region is reduced at the outboard span location (i.e. 75\%) compared to the inboard span location (i.e. 50\%). Additionally, for $k=0.39$, the starting vortex at the trailing edge convects to downstream as free stream velocity increases and forms the first vortex in the wake (the classical starting vortex). As reduced frequency further decreases, the starting vortex is not observed either at the trailing edge or in the wake. Multiple concentrations of vorticity are observed. 

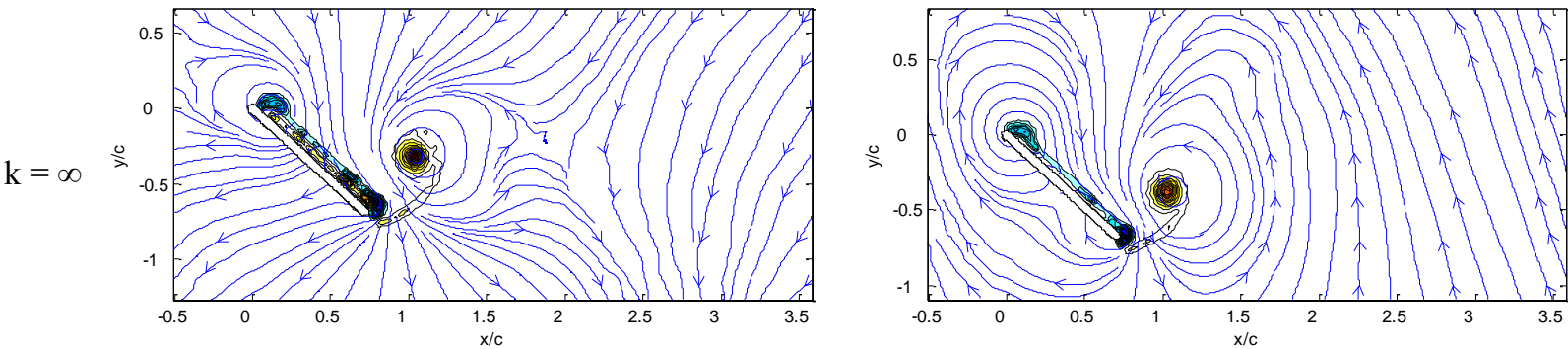

0.394
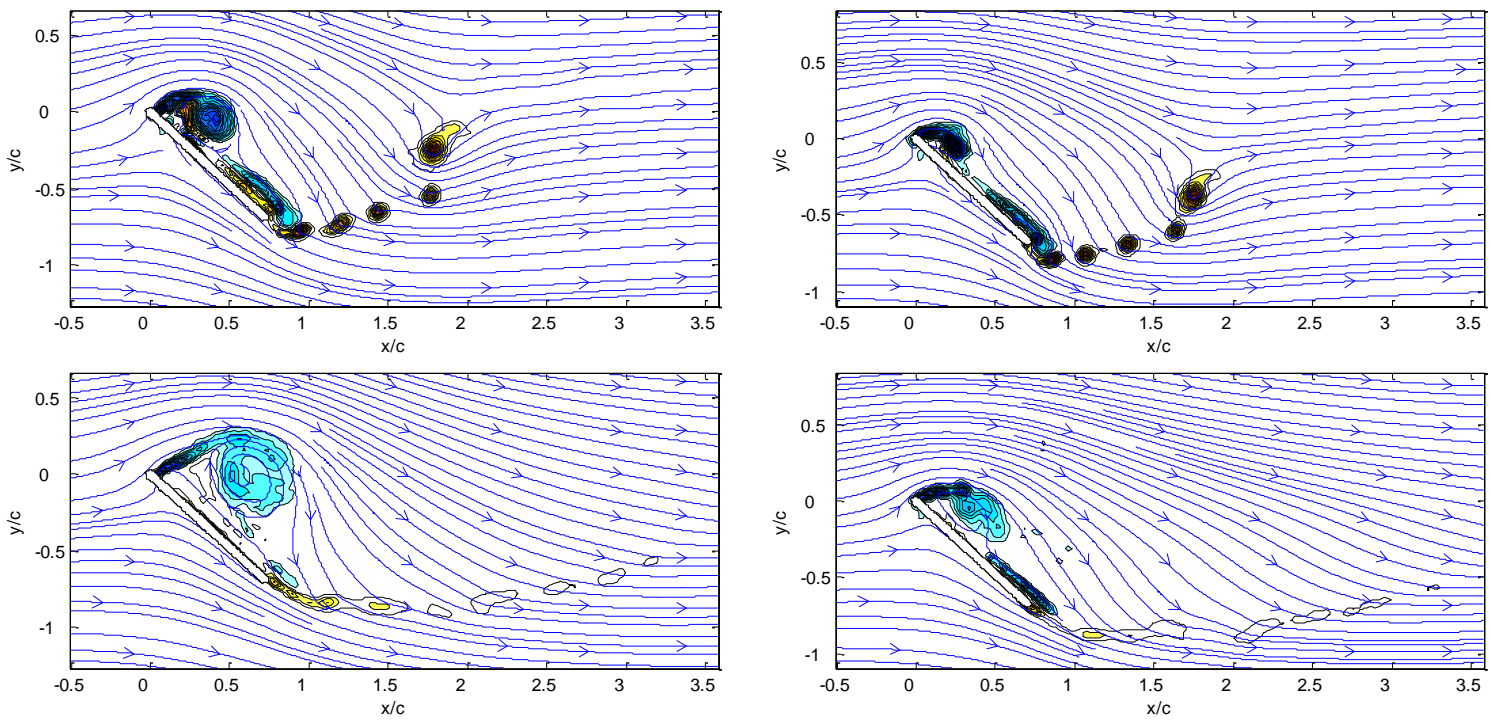

0.132
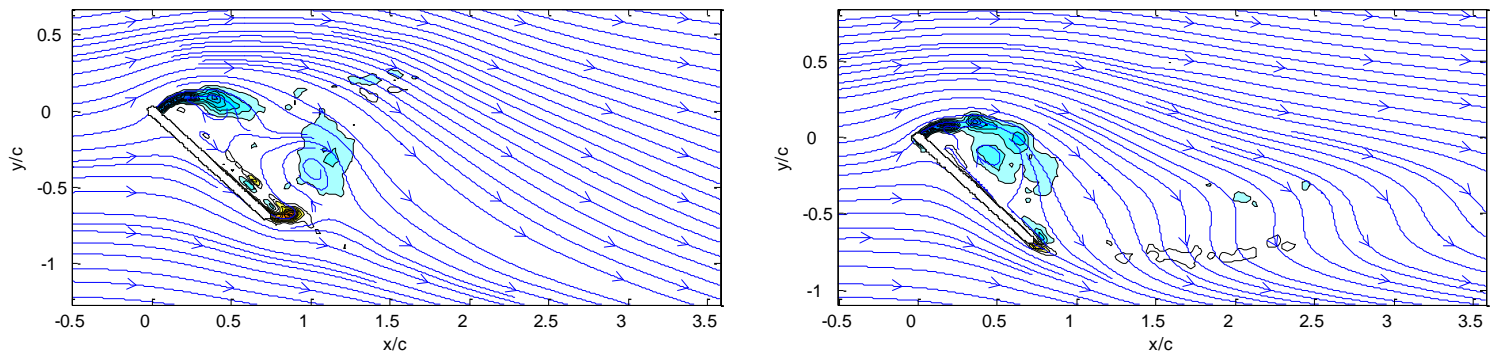

Figure 17. Vorticity fields for leading edge pivot axis at angle of attack 45 degrees and reduced frequency as indicated in the left column. The vorticity field at 50\% span is shown on the left and the vorticity field at $75 \%$ span is shown at the right.

At higher angle of attack, 45 deg, Fig 17 shows that LEV vortices form on the leeward side of the wing for finite reduced frequency. At the inboard span location (i.e. 50\% of span), the LEV grows in size and remains attached as reduced frequency decreases. At $k=0.065$, the LEV is detached and splits in two; meanwhile, the upstream flow at the trailing edge facilities the formation of a trailing edge vortex. However, at outboard span location (i.e. $75 \%$ of span), there is no evidence of the LEV vortex splitting found, which suggests that an arch vortex structure might be present at these flow conditions (Yilmaz and Rockwell ${ }^{13}$ and Visbal ${ }^{14}$ ). The size of the LEV is significantly smaller at the outboard span location and finite reduced frequency. Now considering the formation of the starting vortex, the starting vortex formed at earlier time detaches from the wind surface in the still water, and convects away in the wake at reduced frequency of 0.39 , followed by the formation of several vortices in the wake. Another interesting feature is the pattern of streamlines downstream of the plate in the near wake. It shows almost vertical streamlines at the $75 \%$ spanwise location, which is believed to be associated with a stronger tip vortex. 

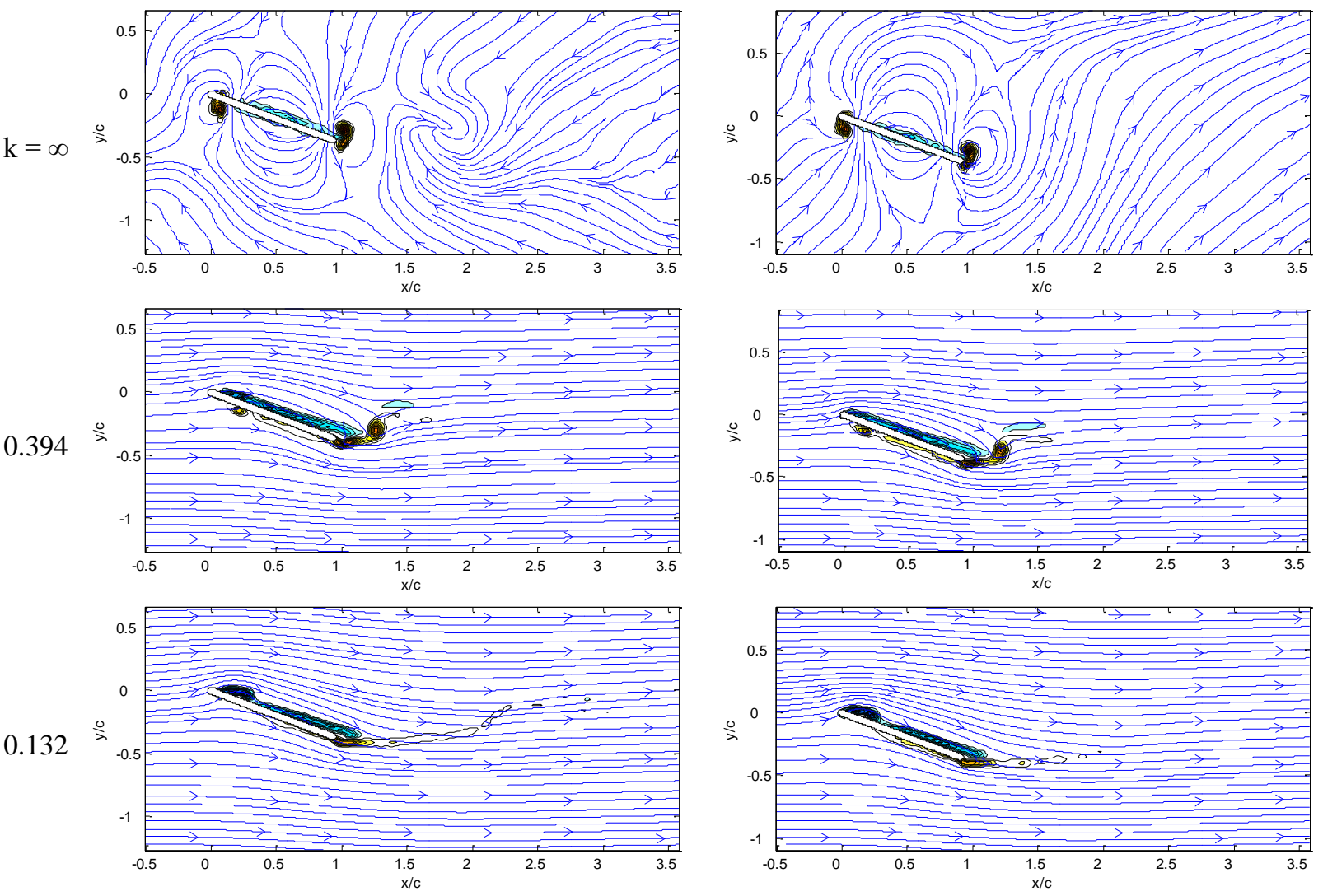

0.132
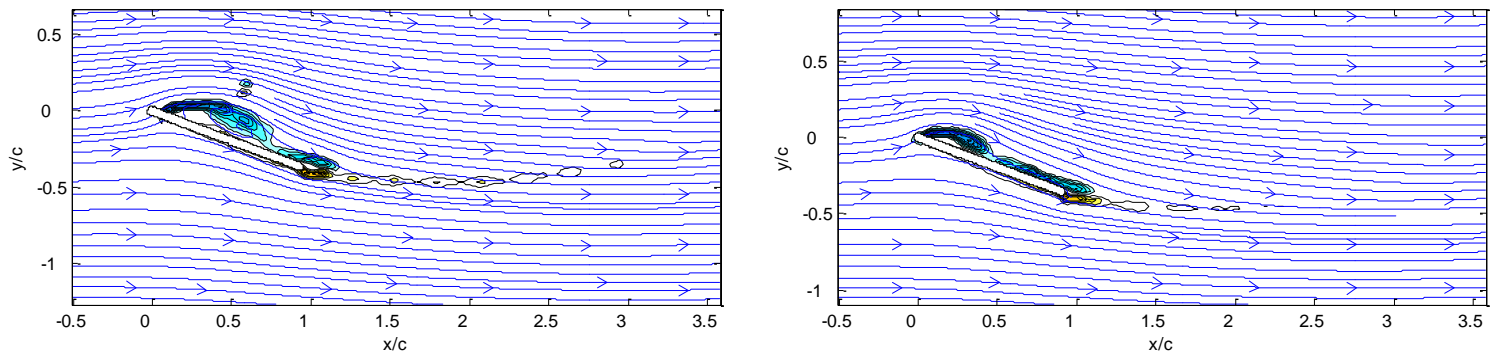

Figure 18. Vorticity fields for mid-chord pivot axis at angle of attack 22.5 degrees and reduced frequency as indicated in the left column. The vorticity field at 50\% span is shown on the left and the vorticity field at $75 \%$ span is shown at the right.

The flow topology for mid-chord pivot axis is shown in Fig. 18 and 19 for angles of attack 22.5 and 45 degrees, respectively. In this case the flow topology is different compared to the leading edge pivot axis. However, there are two common features throughout the pitch ramp phase. First, in the still water (i.e. $k=\infty$ ) the wing motion produces symmetric starting vortices at the leading edge and trailing edge with the same sign of vorticity, both are counterclockwise. These vortices correspond to the starting vortex observed at LE pivot. The size of these vortices is larger at higher angle of attack as shown in Fig. 19. The second feature is that spanwise variation of these vortex patterns is small for reduced frequency above 0.39. At the end of rotation (i.e. 45 degrees) shown in Fig. 19 the vortices remain close to the wing and show similar features compared to the results at 22.5 degrees.

For finite reduced frequency Fig. 18 shows the development of LEV and TEV vortices for different values of $k$ at $22.5^{\circ}$ angle of attack. At $k=0.394$ a starting vortex at the trailing edge is observed in the near wake, and a small starting vortex is found on the windward side of the plate. At lower reduced frequency $k=0.132$ a small LEV forms. At $k=0.065$ the typical LEV develops on the leeward side of the wing at the inboard span location but it is not welldeveloped compared to the leading edge pivot cases. Spanwise variations are small at higher reduced frequency. At the lowest reduced frequency the LEV at 75\% span is significantly smaller than at 50\% span. 

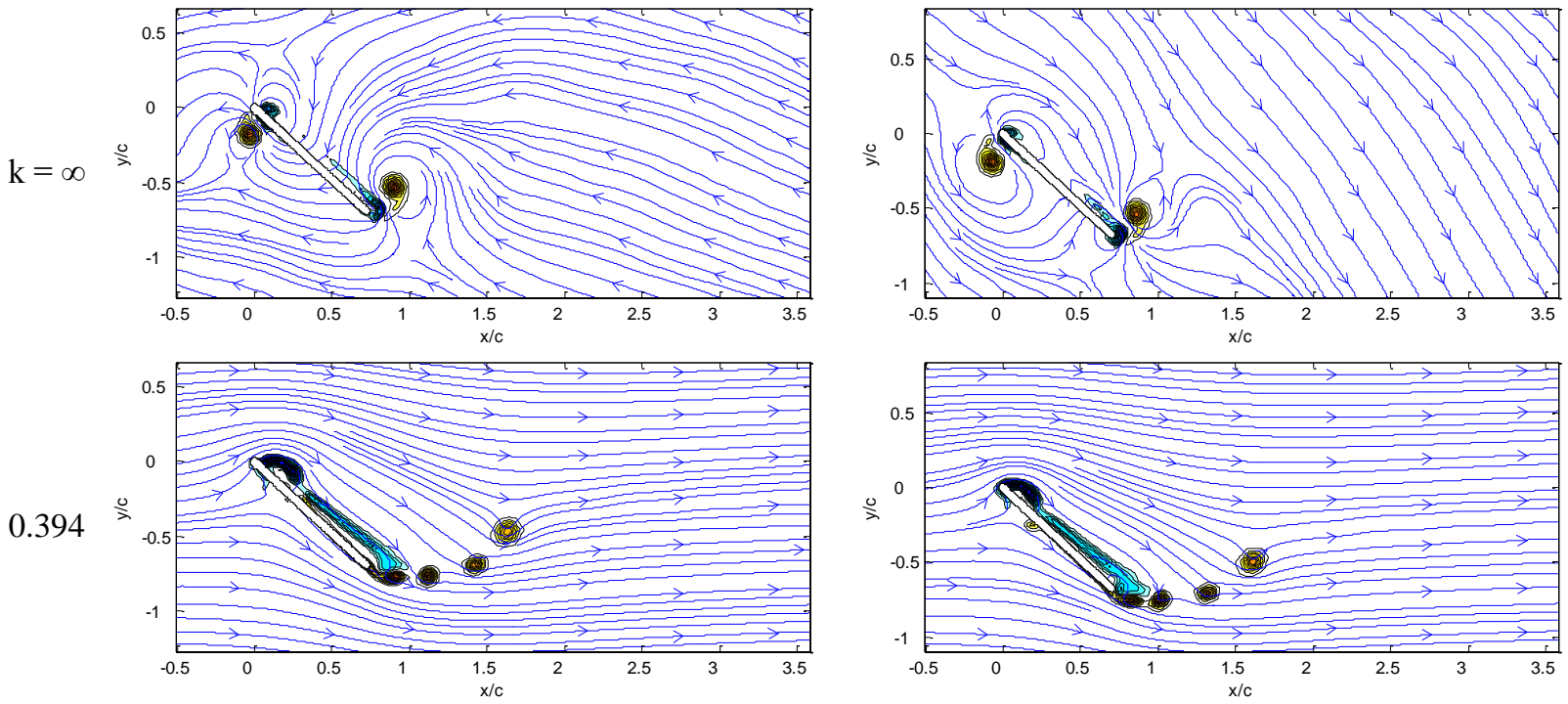

0.394
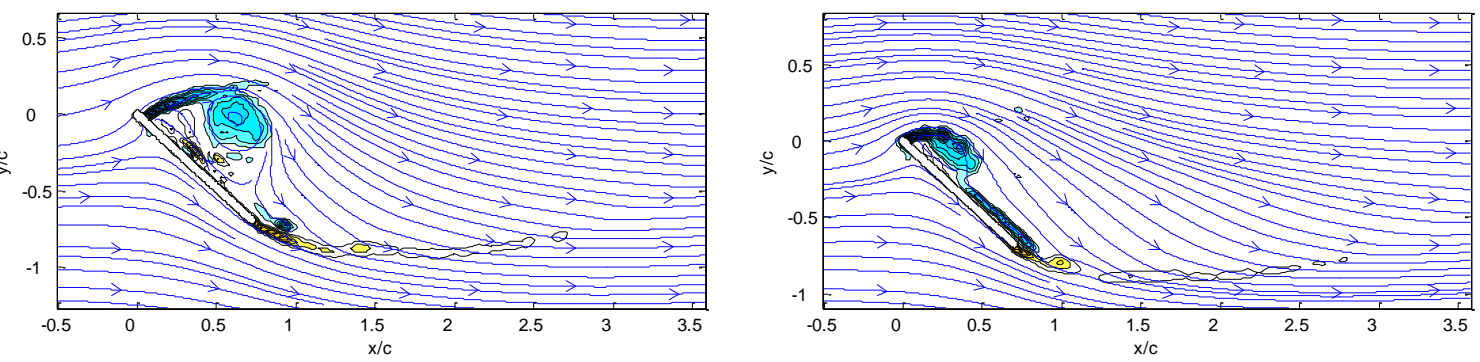

0.132
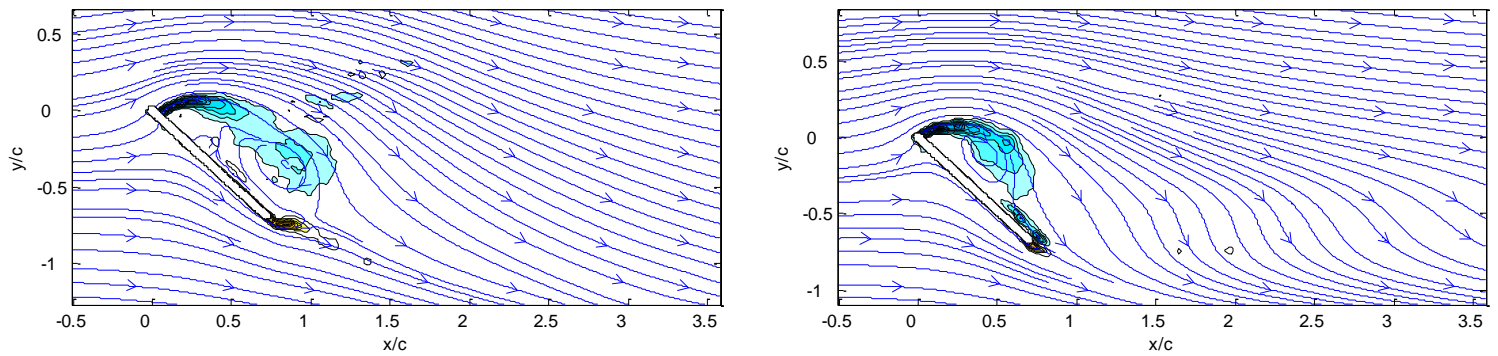

Figure 19. Vorticity fields for mid-chord pivot axis at angle of attack 45 degrees and reduced frequency as indicated in the left column. The vorticity field at 50\% span is shown on the left and the vorticity field at $75 \%$ span is shown at the right.

The flow topology for mid-chord pivot axis at the end of the pitch ramp motion (i.e. 45 degrees) is shown in Fig. 19. The flow field shows similar features as the cases at the leading edge pivot axis, shown in Fig. 17. For finite reduced frequency a LEV is more developed as reduced frequency decreases. This LEV is attached for reduced frequencies greater than 0.065 . At $k=0.065$ the LEV is detached forming a small trailing edge vortex. The vorticity distribution at mid-span location shows two peaks which suggest splitting of the LEV. As for the leading edge pivot, LEV splitting is not observed at the outboard span location, which again would be an indication of the presence of an arch vortex at these conditions. Comparing the flow structure at $50 \%$ and $75 \%$ spanwise locations shows similar features as for the leading edge pivot cases. The LEV size is significantly smaller at the $75 \%$ span. A small starting vortex formed at leading edge is advected along the windward side of the wing at high reduced frequency (i.e. $k=$ 0.394). The starting vortex formed at the trailing edge of the wing moves away from the trailing edge with increasing free stream velocity and splits into several smaller vortices. The number of the vortices formed in the wake is less than what was found at leading edge pivot. These wake vortices decreased in strength as the reduced frequency is decreased. The strong downwash shown by the streamline pattern is also present for mid-chord pivot. 

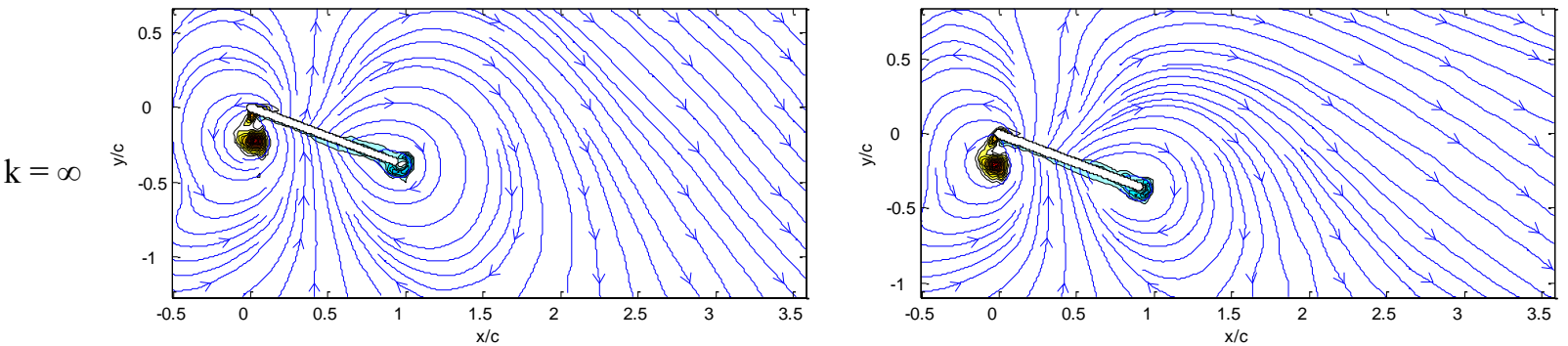

0.394
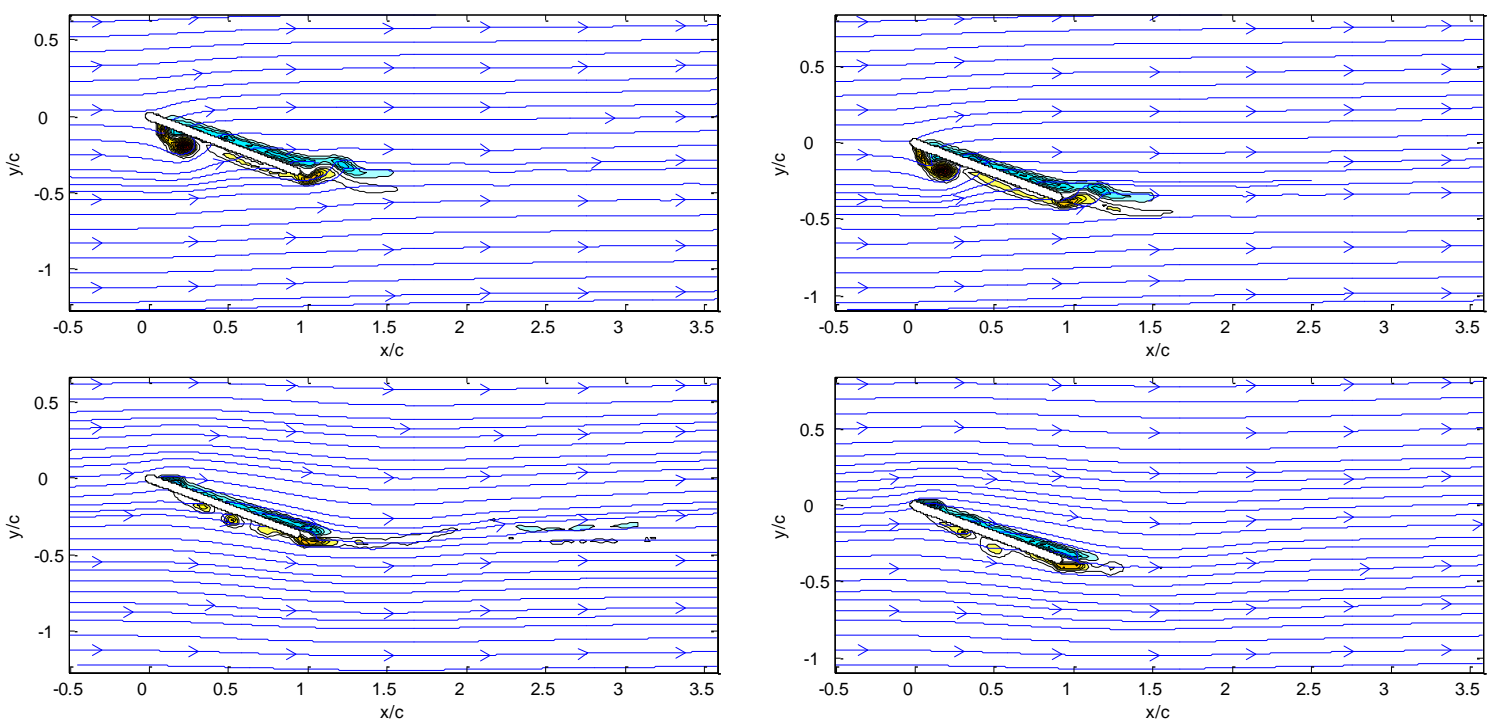

0.132
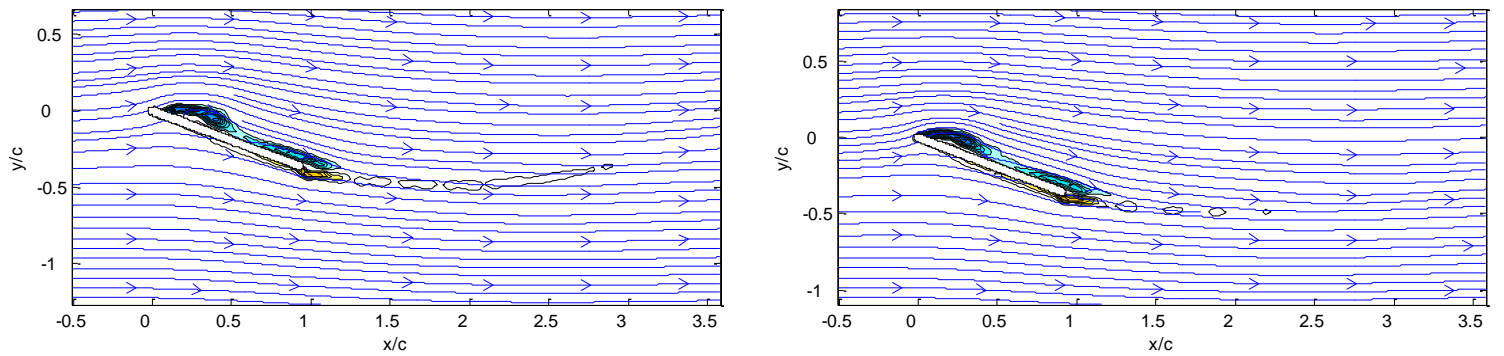

Figure 20. Vorticity fields for trailing edge pivot axis at angle of attack 22.5 degrees and reduced frequency as indicated in the left column. The vorticity field at 50\% span in shown on the left and the vorticity field at $75 \%$ span is shown at the right.

The flow topology for trailing edge pivot axis is shown in Fig. 20 and 21 for angles of attack 22.5 and 45 degrees, respectively. The two main features noted for the other pivot axes are also present in this case during the pitch ramp phase. First, in the still water (i.e. $k=\infty$ ), the wing motion produces a starting vortex at the leading edge similar to the starting vortices observed at the other two pivot locations. Secondly, the difference between the vorticity distributions at the two spanwise locations is small for reduced frequency $k>0.39$. This starting vortex at the leading edge is advected along the windward side of the wing as the free stream velocity increases. Because it forms on the pressure side of the wing and has opposite sign of vorticity compared to the LEV, the evolution of the flow and forces in this case is different.

For finite reduced frequency Fig. 20 shows the development of vorticity in the flow at $22.5^{\circ}$ angel of attack for different values of $\mathrm{k}$. At $k=0.394$ the starting vortex forms, and LEV or TEV vortices are not observed. Similarly, at $k=0.132$ there is only a small concentration of vorticity at the leading edge, and the starting vortex is advected downstream on the pressure side of the plate and splits into several smaller vortices. At $k=0.065$ there is a small region of separated flow at the leading edge at the 50\% spanwise location, this region is smaller at outboard span location. The spanwise variations of flow features are small at this lower angle of attack for higher reduced frequency 

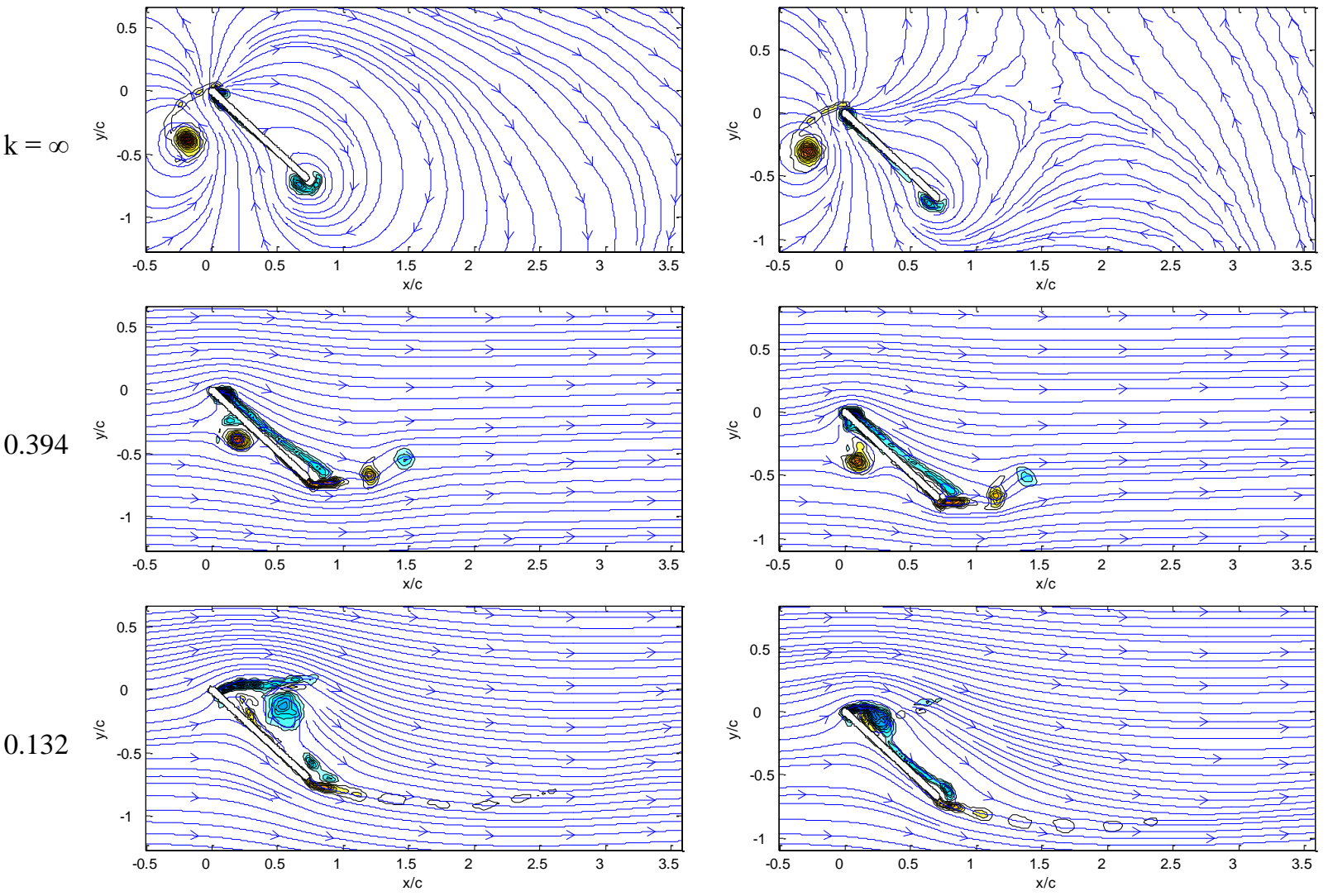

0.132
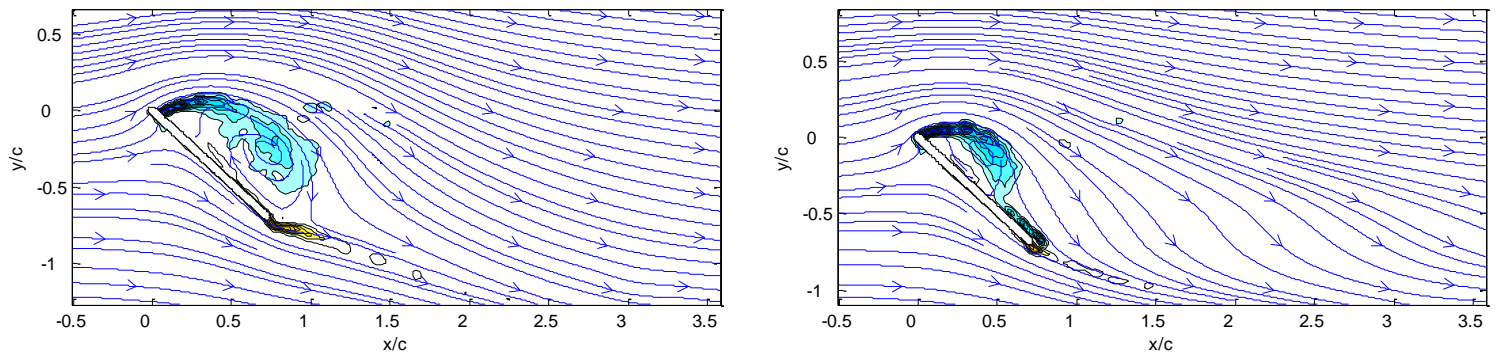

Figure 21. Vorticity fields for trailing edge pivot axis at angle of attack 45 degrees and reduced frequency as indicated in the left column. The vorticity field at 50\% span is shown on the left and the vorticity field at $75 \%$ span is shown at the right.

The flow topology for trailing edge pivot axis at the end of the pitch up motion (45 degrees) is shown in Fig. 21. At high reduced frequency the flow topology is fundamentally different compared to other pivot axis locations. At $k$ $=0.394$ the starting vortex at the leading edge moves downward but still remains on the pressure side of the wing at the end of the wing rotation. TEV vortices form in the near wake; however, the first vortex has opposite sign of vorticity compared to the starting vortex for the other two pivot locations. At lower reduced frequency the starting vortex at the leading edge is not observed and the development of the LEV is observed. Formation of the LEV is significantly delayed compared to cases at leading edge and mid-chord pivot axes. For $k=0.065$ the LEV is attached and LEV splitting is not present at the inboard span location. Comparing the LEV at different span locations, the inboard span location shows much larger structure than the outboard span location. Also at $k=0.065$ the streamlines indicate strong downwash in the near wake region, similar to the flow for the other pivot axes.

\section{Conclusion}

Unsteady aerodynamic force and PIV velocity measurements have been obtained for a flat plate wing with aspect ratio 4 during a pitch-up motion up to 45 degrees. Changes in pitch rate and free stream velocity are considered resulting in a parameter space with reduced frequencies in the range $0.022-0.394$. Also the case of $R e=$ 0 (i.e. $k=\infty$ ) has been documented to gain additional insight on the nature of non-circulatory effects. The main conclusions of this investigation are as follows: 
From the force measurements,

1. In all cases the force coefficients increase during the pitch-up portion of the motion and decrease until they reach the steady state condition.

2. Force oscillations are observed during the transient in the hold phase $\left(t / t_{c} \sim 4\right)$ consistent with incipient vortex shedding, but disappear after approximately 30 convective times before the steady state condition is reached.

3. Non-circulatory apparent mass effects are found for reduced frequency greater than 0.066 and for leading and trailing edge pivot axes. These effects are confined to the non-zero acceleration region at the start and the end of the wing rotation.

4. Non-circulatory apparent mass effects are small at lower frequency $k=0.065$ and for mid-chord pivot axis.

5. Rotation rate effects are observed in the constant pitch rate region for reduced frequency $k>0.022$

6. For leading edge pivot axis non-circulatory effects and rotation rate effect combine to produce very large force coefficients, well above estimates based on the lifting line theory.

7. For trailing edge pivot axis non-circulatory effects and rotation rate effects oppose each other resulting in lower force coefficients below the lifting line estimates.

8. For reduced frequency below $k=0.022$ the flow is quasi steady and the lifting line theoretical results provide good estimates of the lift coefficients for angles of attack below the steady stall angle, approximately 20 degrees.

9. For reduced frequency below $k=0.022$ the drag coefficient estimates based on the lifting line theory for induced drag are a factor of 2 below measured results. This is attributed to the failure of accounting the low leading edge suction force on the present thin flat plate.

From the PIV images,

10. PIV measurements show formation of LEV and TEV vortices at the end of the pitch up motion, but the rate of development depends on pivot axis location and reduced frequency.

11. PIV measurements also show important differences in flow topology for different pivot axis due to the formation of starting vortices. Different starting vortex is produced by rotating the wing at zero free stream velocity. For leading edge pivot axis the starting vortex is formed at the trailing edge. For mid-chord pivot axis starting vortices are formed at the leading edge and the trailing edge. For trailing edge pivot axis the starting vortex forms at the leading edge.

12. Formation of a starting vortex at the leading edge delays the development of the LEV.

13. Significant spanwise variation of the LEV size is found for lower reduced frequency. The measured changes in flow topology at low reduced frequency are consistent with formation of an arch vortex on the leeward side of the plate.

\section{Acknowledgments}

The authors would like to thank Drs. Michael Ol and Kenneth Granlund of the Air Force Research Laboratory (AFRL) for many fruitful discussions of the research reported in this paper. The work was sponsored in part by the Air Force Office of Scientific Research's Multidisciplinary University Research Initiative (MURI), contract number FA9550-07-1-0547 and by the Michigan/AFRL Collaborative Center in Aeronautical Sciences. 


\section{Appendix A}
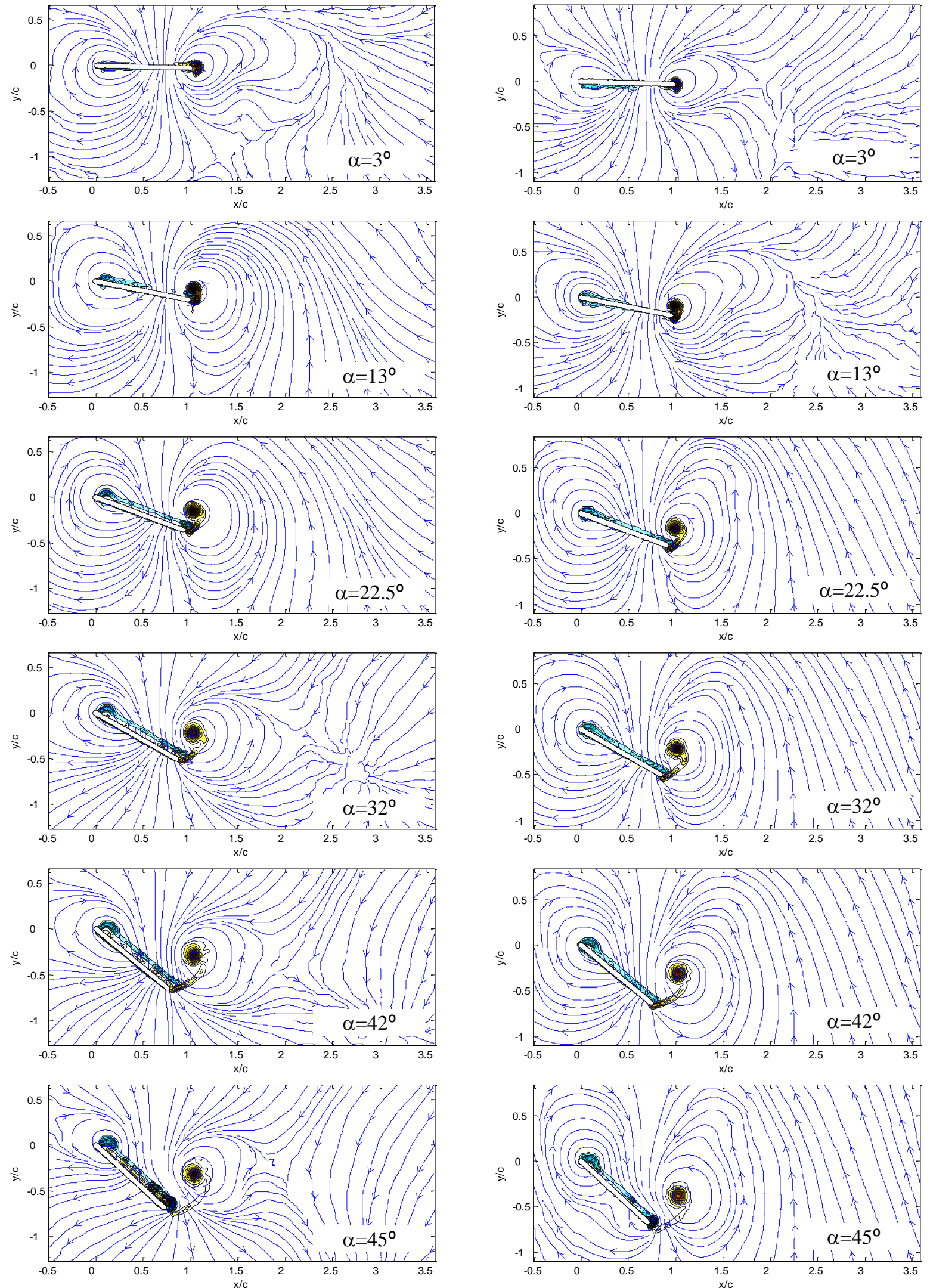

Figure A.1. Flow evolution for $k=\infty$ at leading edge pivot (left) $50 \%$ of span (right) $75 \%$ of span 23

American Institute of Aeronautics and Astronautics 

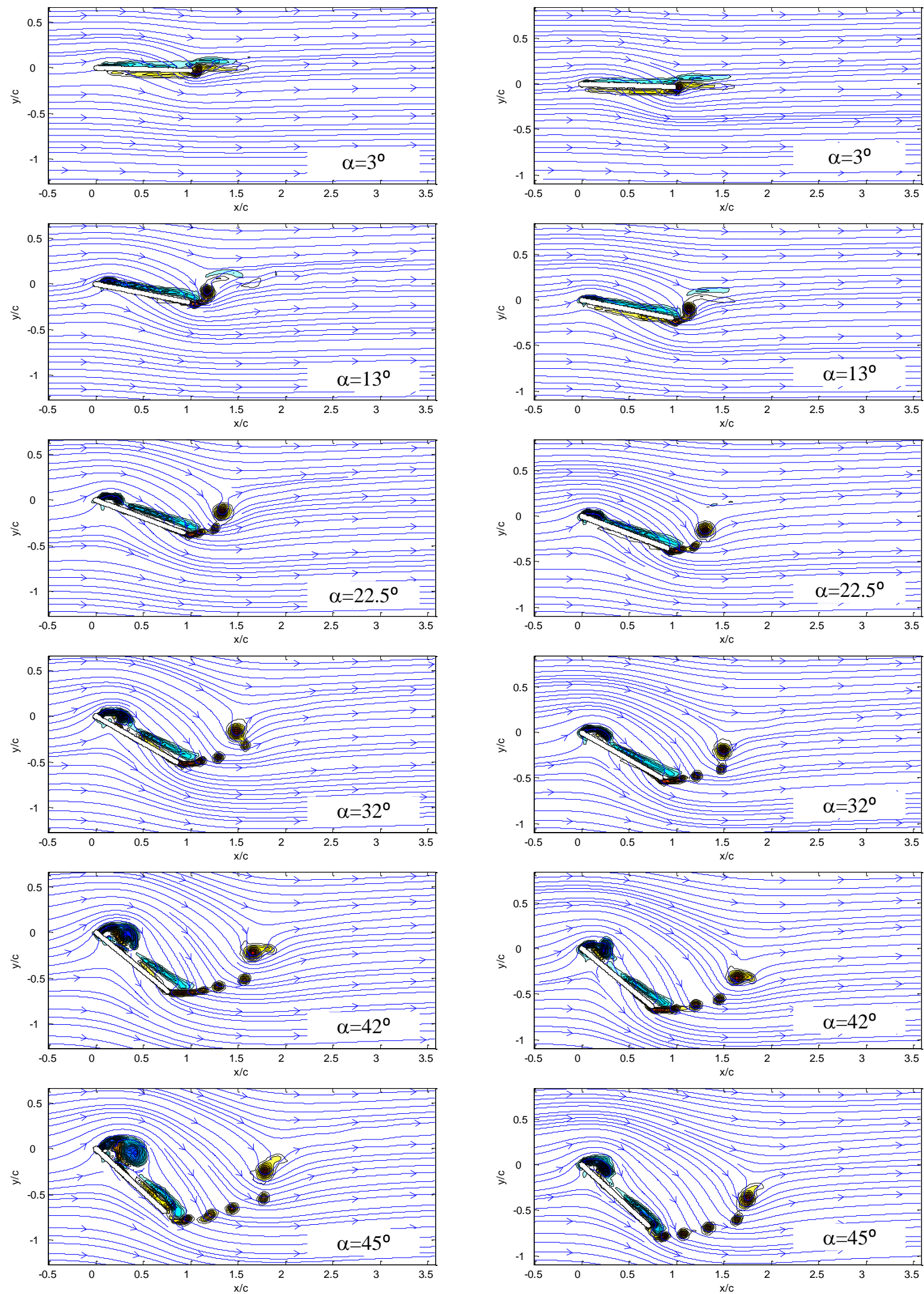

Figure A.2. Flow evolution for $k=0.394$ at leading edge pivot (left) $\mathbf{5 0 \%}$ of span (right) $\mathbf{7 5 \%}$ of span 

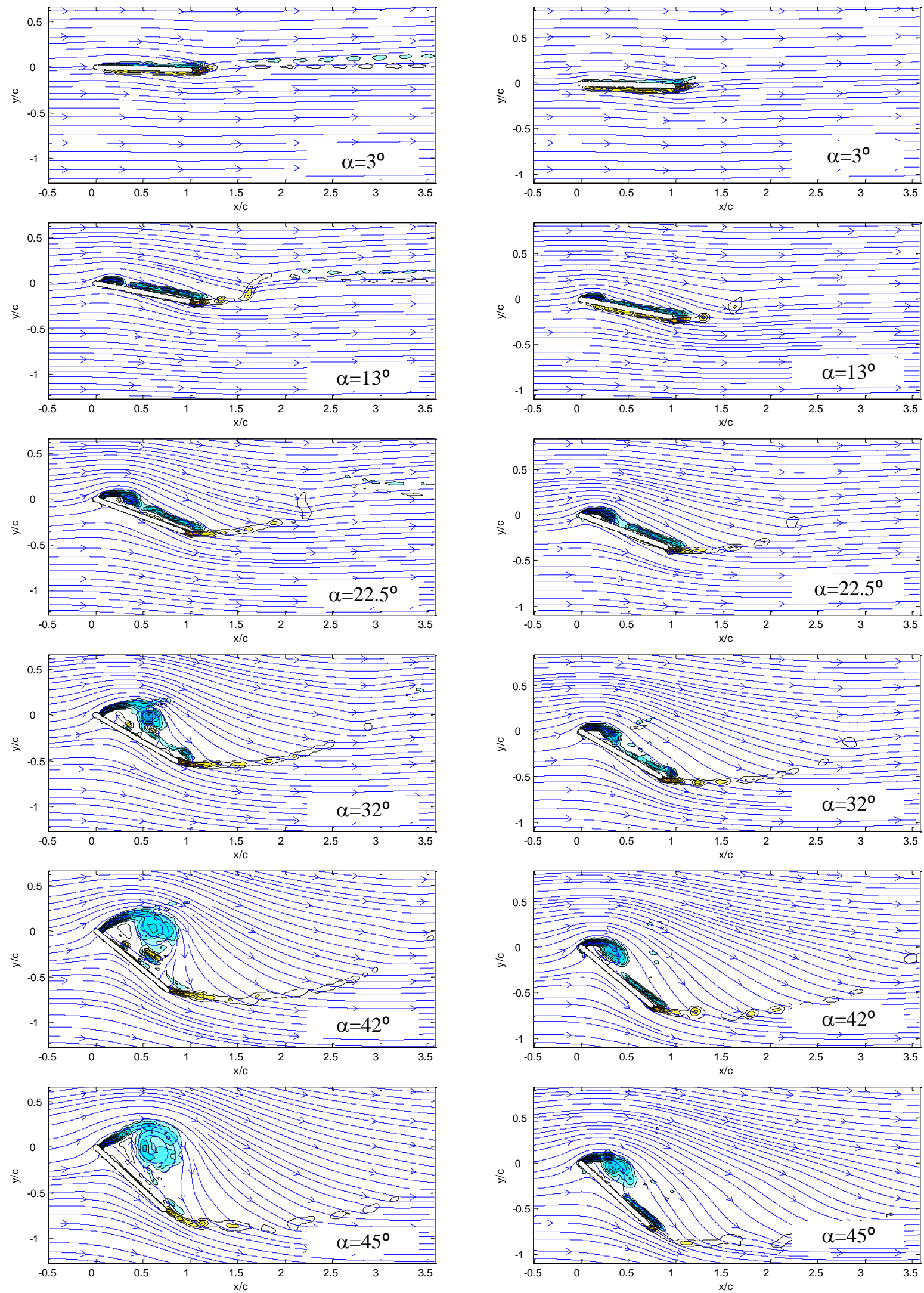

Figure A.3. Flow evolution for $k=0.132$ at leading edge pivot (left) $50 \%$ of span (right) $75 \%$ of span 

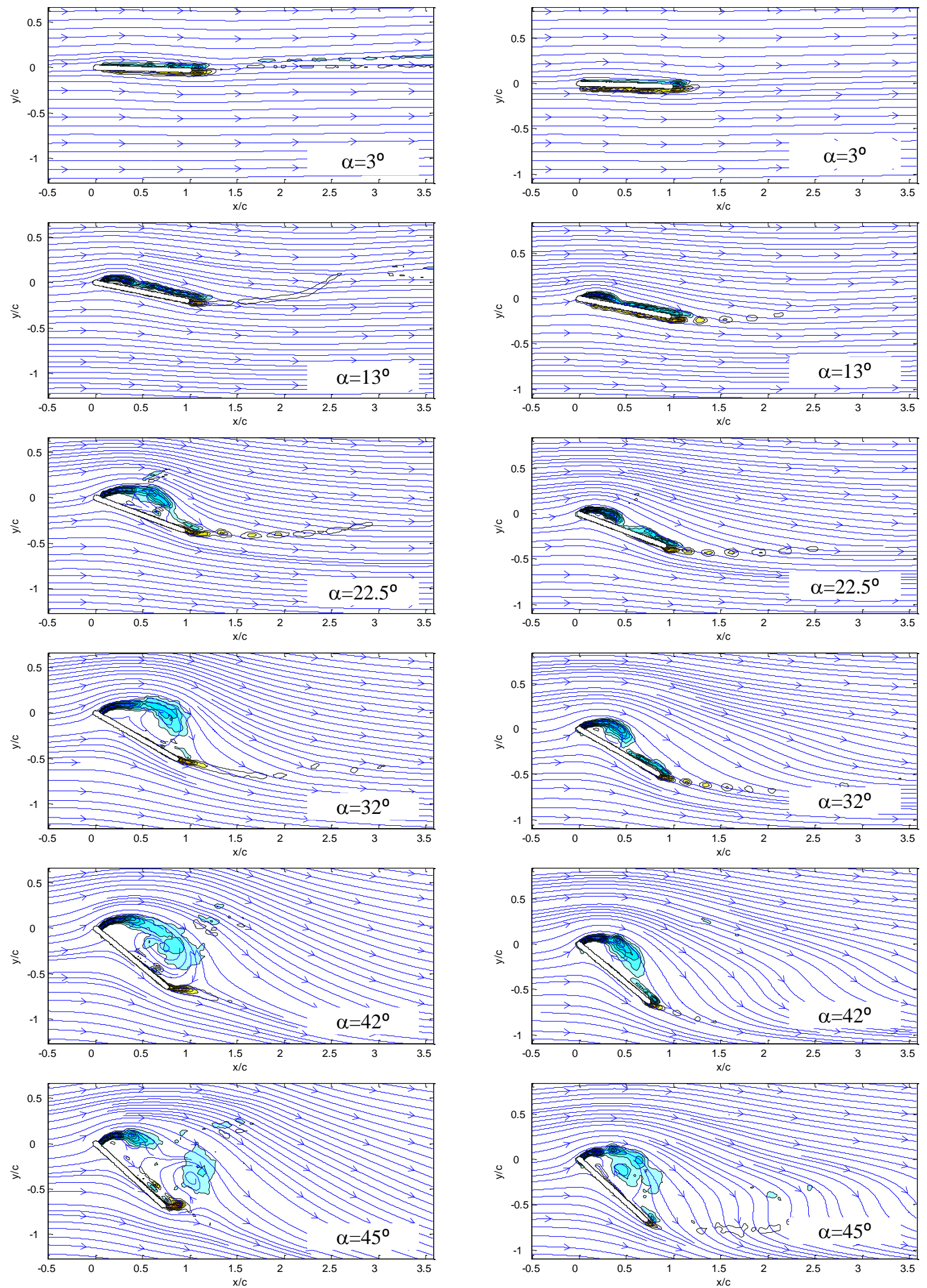

Figure A.4. Flow evolution for $k=\mathbf{0 . 0 6 5}$ at leading edge pivot (left) $\mathbf{5 0 \%}$ of span (right) $\mathbf{7 5 \%}$ of span 

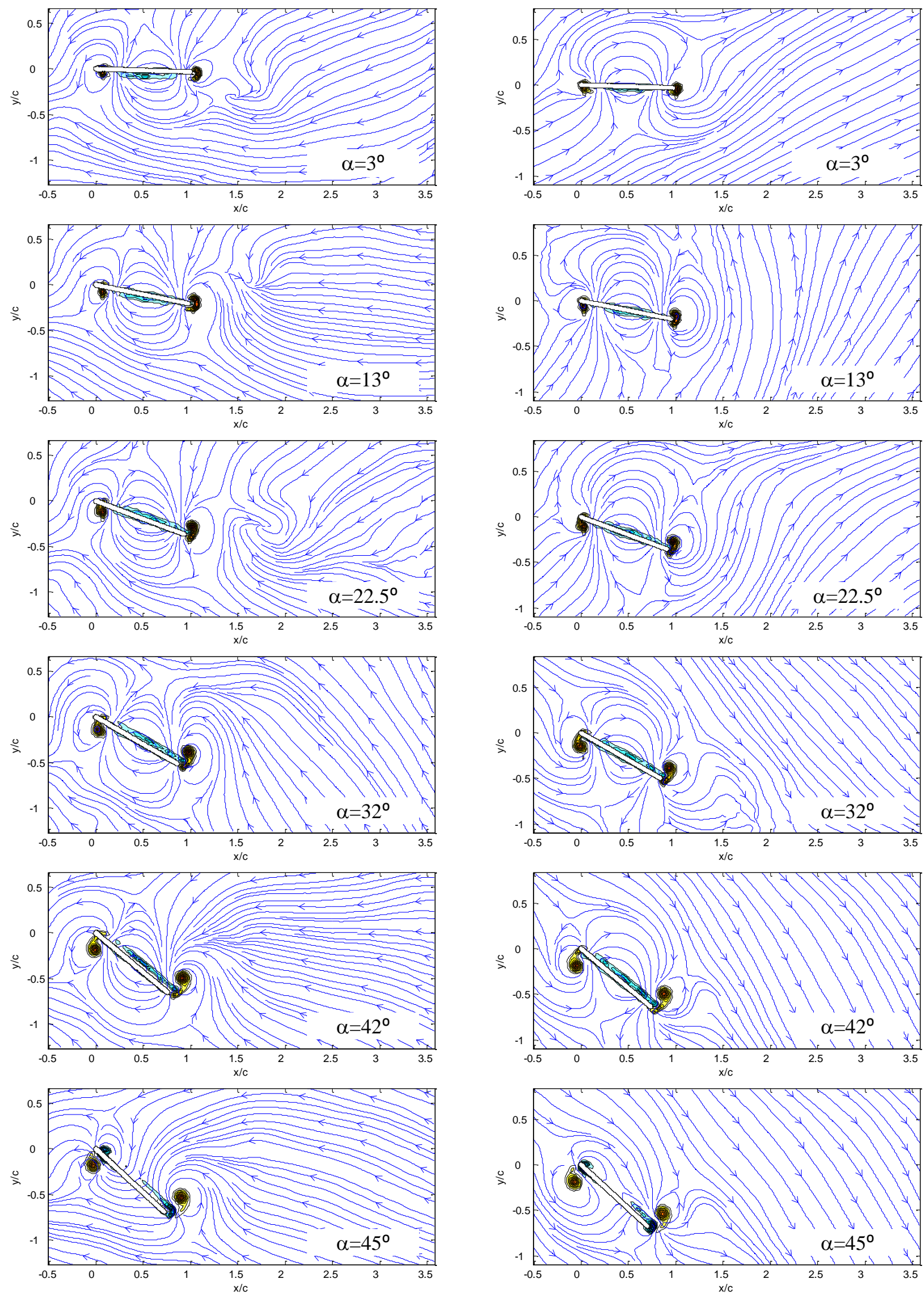

Figure A.5. Flow evolution for $k=\infty$ at mid-chord pivot (left) $50 \%$ of span (right) $75 \%$ of span 

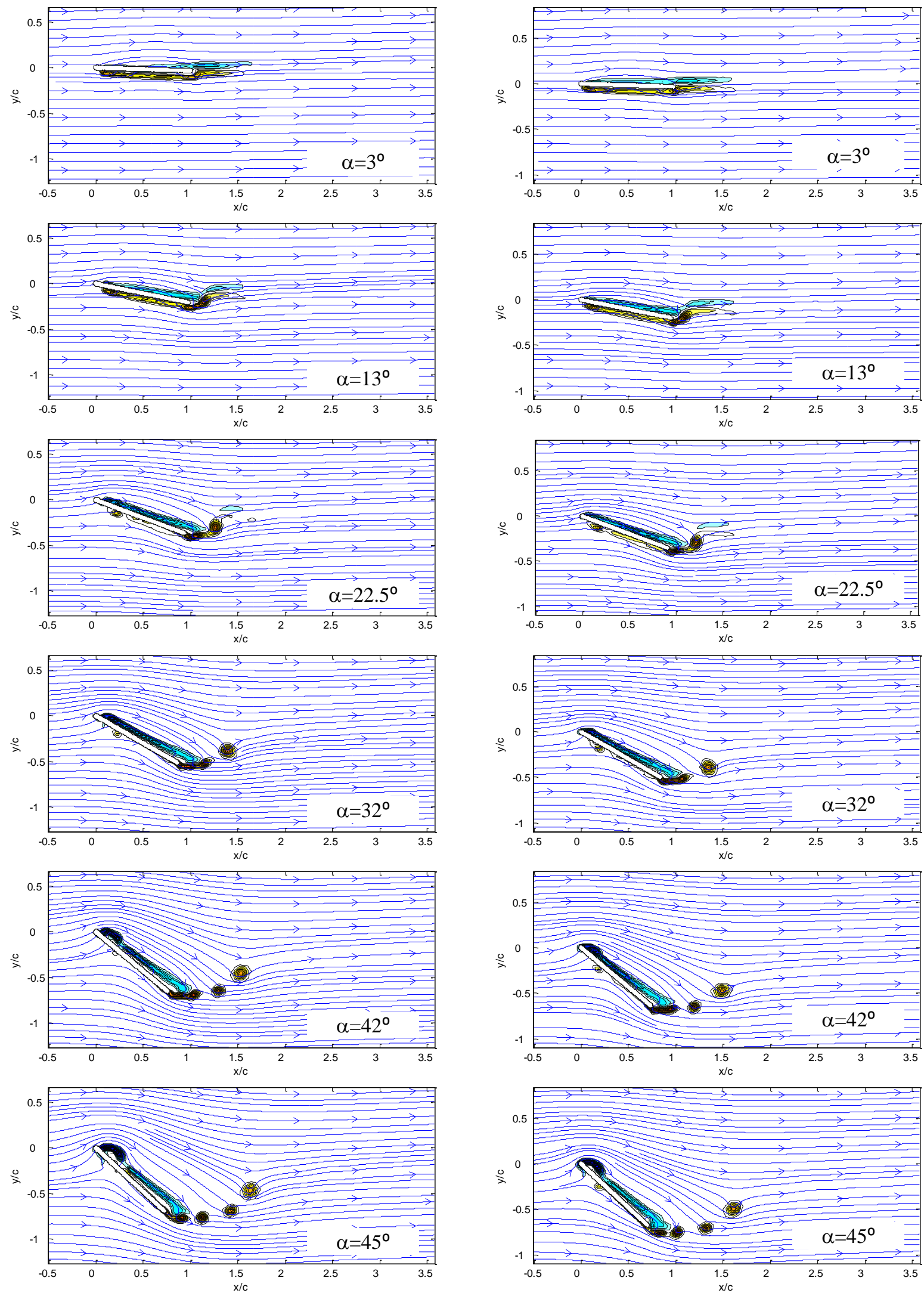

Figure A.6. Flow evolution for $k=0.394$ at mid-chord pivot (left) $50 \%$ of span (right) $75 \%$ of span 

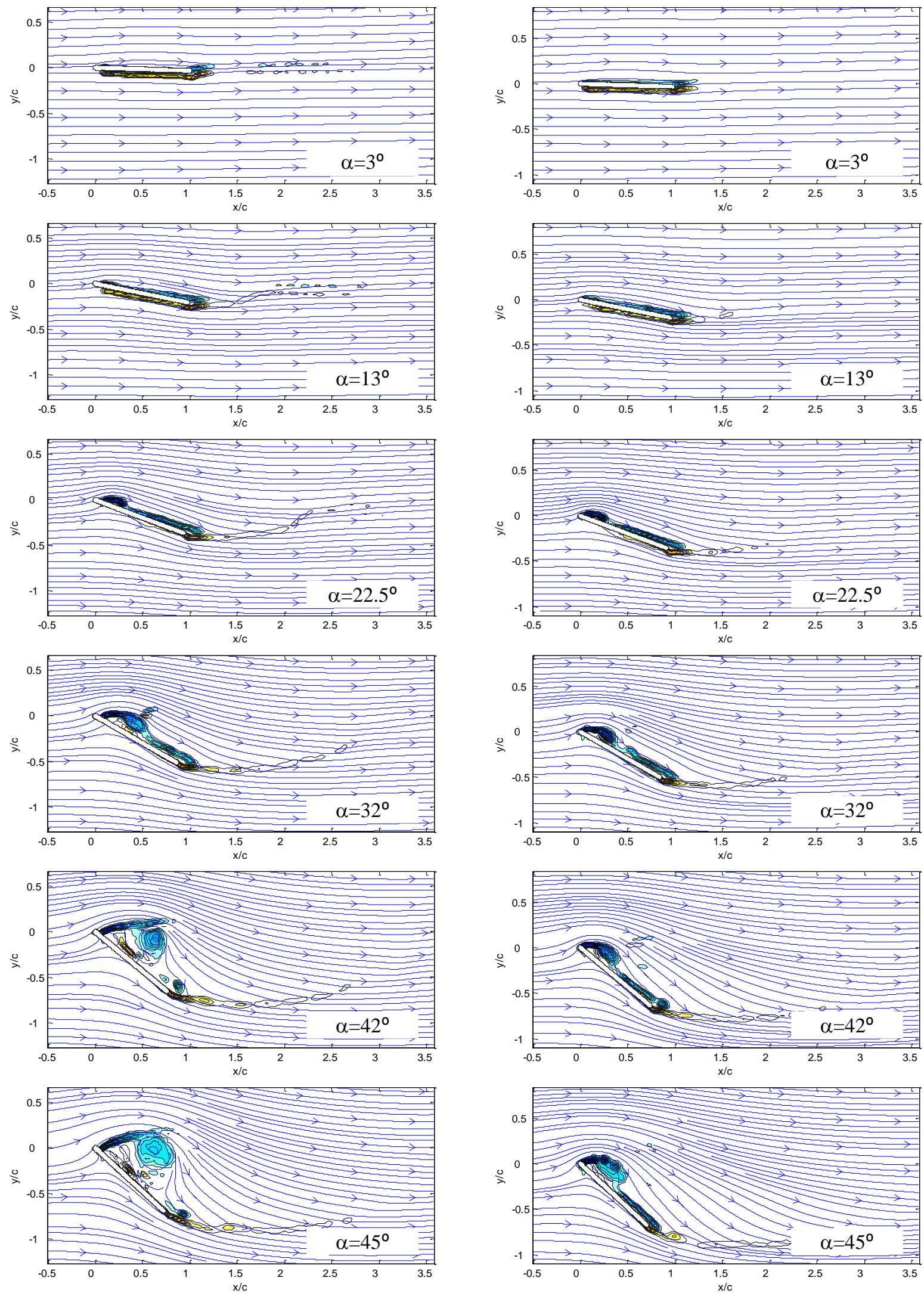

Figure A.7. Flow evolution for $k=0.132$ at mid-chord pivot (left) $50 \%$ of span (right) $75 \%$ of span 

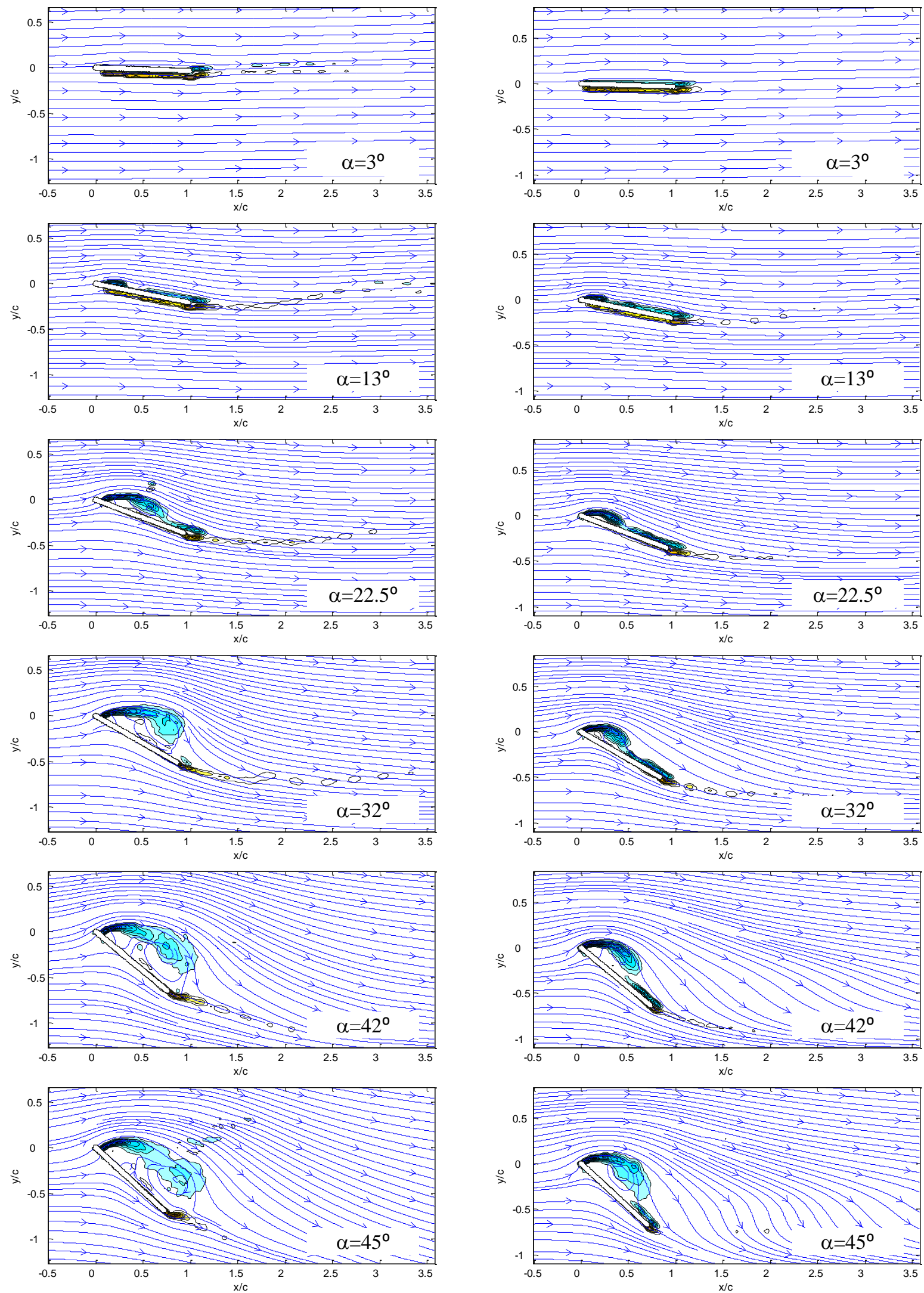

Figure A.8. Flow evolution for $k=0.065$ at mid-chord pivot (left) $50 \%$ of span (right) $75 \%$ of span 

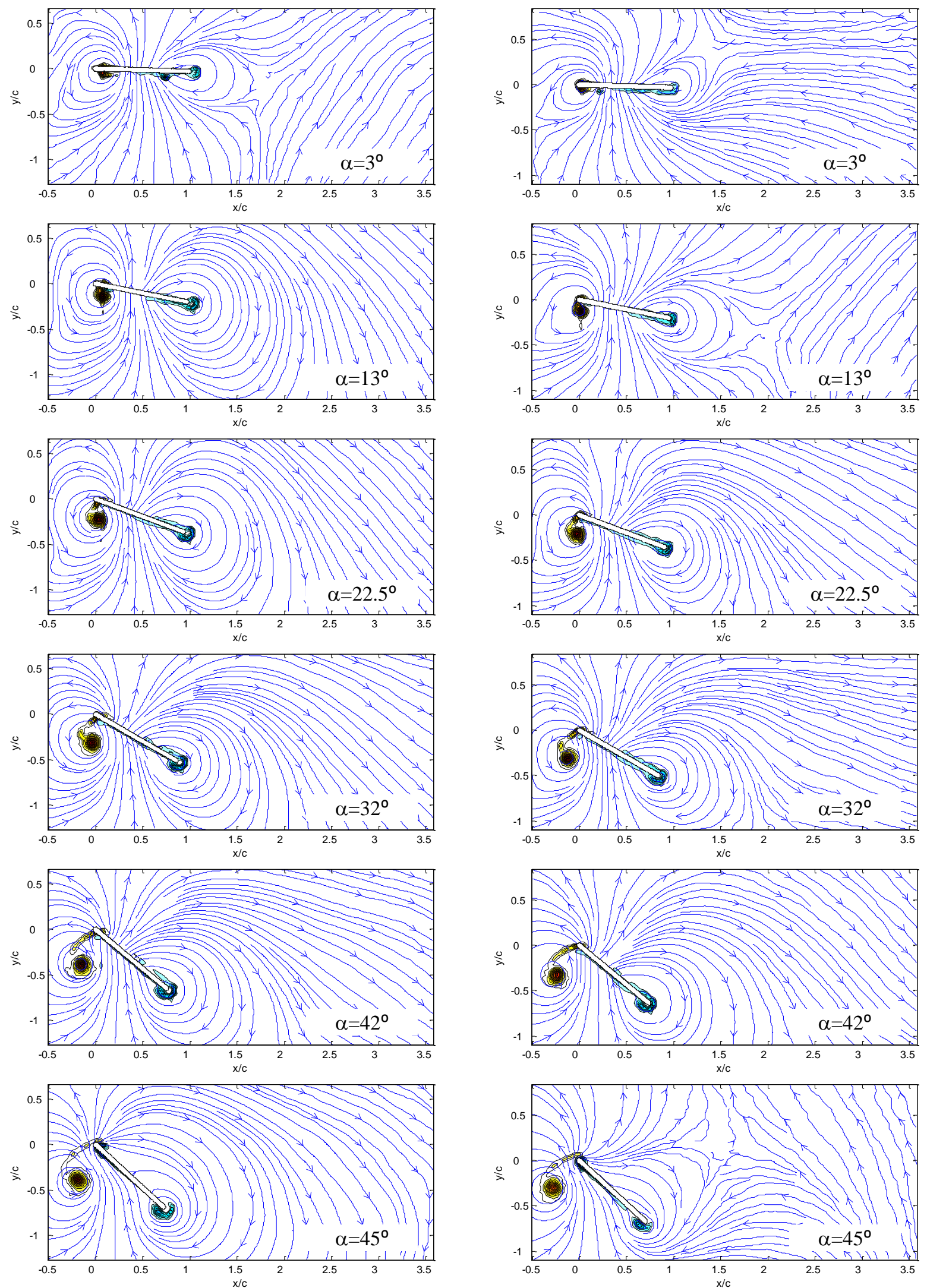

Figure A.9. Flow evolution for $k=\infty$ at trailing edge pivot (left) $50 \%$ of span (right) $75 \%$ of span 

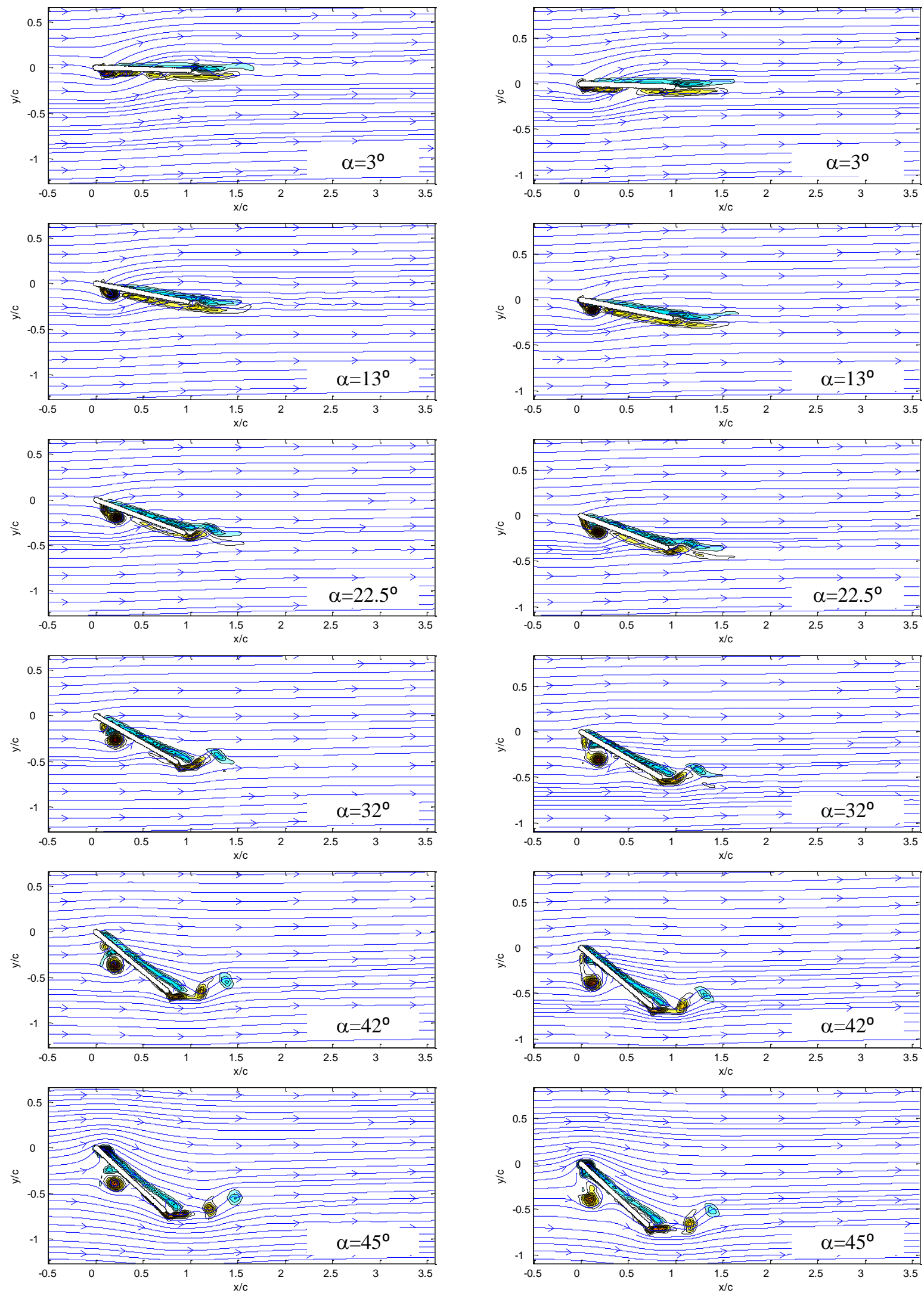

Figure A.10. Flow evolution for $k=0.394$ at trailing edge pivot (left) $50 \%$ of span (right) $\mathbf{7 5 \%}$ of span 

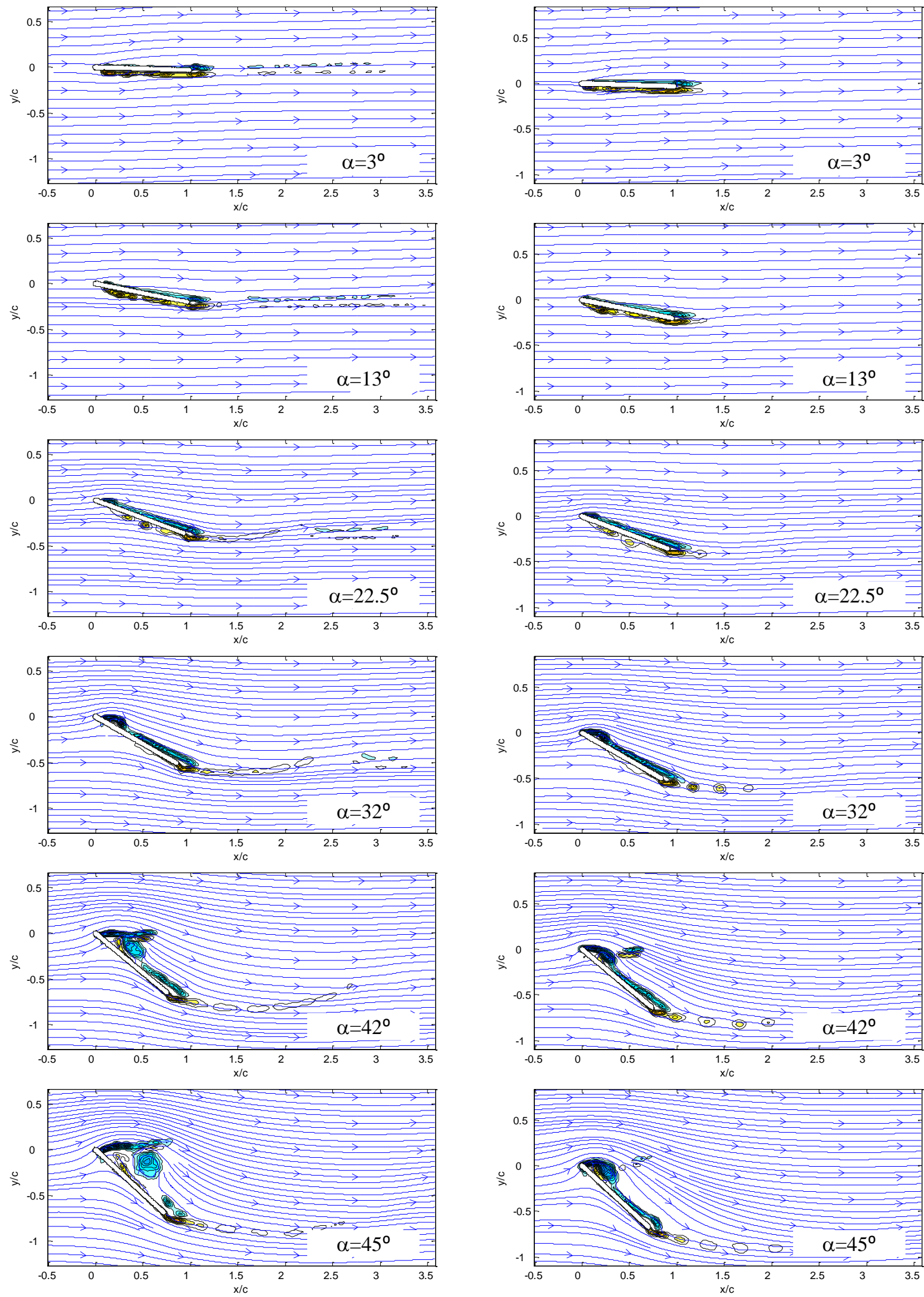

Figure A.11. Flow evolution for $k=0.132$ at trailing edge pivot (left) $50 \%$ of span (right) $75 \%$ of span 

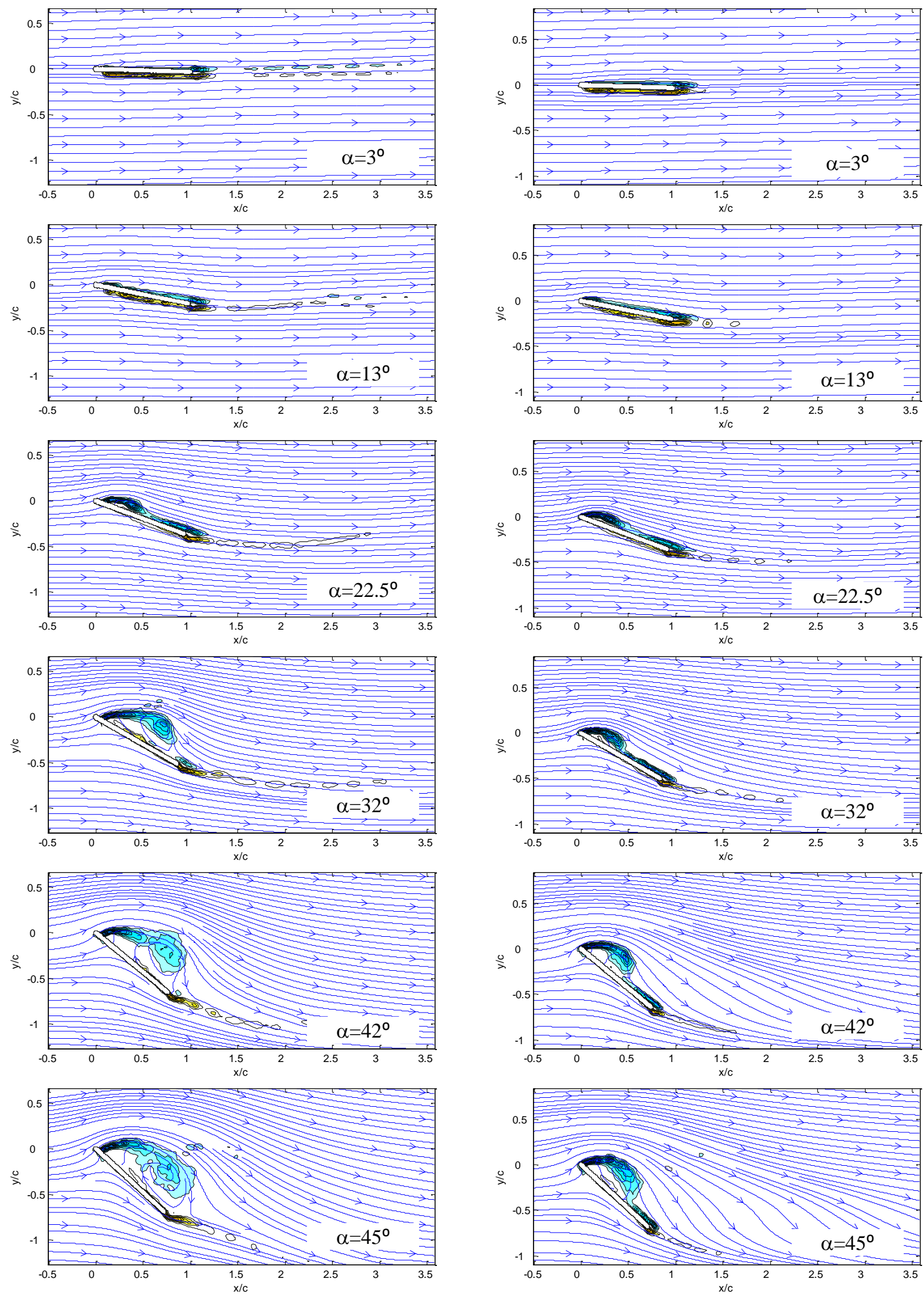

Figure A.12. Flow evolution for $k=0.065$ at trailing edge pivot (left) $50 \%$ of span (right) $\mathbf{7 5 \%}$ of span 


\section{References}

${ }^{1}$ Grandlund, K., Ol, M.V., Garmann, D., Visbal, M. and Bernal, L.P. "Experiments and computations on abstraction of perching", 28th AIAA Applied Aerodynamics Conference, 28 June - 1 July 2010, Chicago, Illinois, AIAA Paper $2010-4943$.

${ }^{2}$ Ramesh, K., Gopalarathnam, A., Edwards, J.R., Ol, M.V. and Granlund, K. "Theoretical, Computational and Experimental Studies of a Flat Plate Undergoing High-Amplitude Pitching Motion" 49th AIAA Aerospace Sciences Meeting including the New Horizons Forum and Aerospace Exposition, 4 - 7 January 2011, Orlando, Florida, AIAA Paper 2011-217.

${ }^{3}$ Baik, Y.S., Aono, H., Rausch, J.M., Bernal, L.P., Shyy, W. and Ol, M.V. "Experimental Study of a Rapidly Pitched Flat Plate at Low Reynolds Number," $40^{\text {th }}$ AIAA Fluid Dynamics Conference and Exhibit, 28 June - 1 July 2010, Chicago, Illinois, AIAA Paper 2010-4462.

${ }^{4}$ Yu, H.T., Bernal, L.P. and Morrison, C., "Experimental Investigation of Pitch Ramp-Hold-Return Motion of Flat Plates at Low Reynolds Number", 50th AIAA Aerospace Science Meeting including the New Horizons Forum and Aerospace Exposition, AIAA 2012-51.

${ }^{5}$ Harper, P.W. and Flanigan, R.E., "The Effect of Change of Angle of Attack on the maximum Lift of a small Model", NACA TN 2061, March 1950

${ }^{6}$ McCroskey, W.J. and Philippe, J.J., "Unsteady Viscous Flow on oscillating Airfoils", AIAA Journal, Vol. 13, No. 1, Jan. 1975.

${ }^{7}$ Currier, J.M. and Fung, K.Y., “Analysis of the Onset of Dynamic Stall”, AIAA Journal, Vol. 30, No. 10, October 1992.

${ }^{8}$ Ellington, C.P., Berg, C.V.D., Willmott, A.P., and Thomas, A.L.R., "Leading Edge Vortices in Insect Flight", Nature (London), Vol. 384, 1996, pp. 626-630.

9 Birch, J.M. and Dickinson, M.H., "Spanwise Flow and the Attachment of the Leading Edge Vortex on Insect Wings", Nature (London), Vol. 412, 2001, pp. 729-733.

${ }^{10}$ Shyy, W. and Liu, H., "Flapping Wings and Aerodynamic Lift: The Role of Leading Edge Vortices", AIAA Journal, Vol. 45, No. 12, Dec. 2007.

${ }^{11}$ Eldredge, J. D. and Wang, C., “A Computational Study of a Canonical Pitch-up, Pitch-down Wing Maneuver”, 39th AIAA Fluid Dynamics Conference, AIAA 2009-3687.

${ }^{12}$ Anderson, Fundamentals of Aerodynamics, $5^{\text {th }}$ ed., McGraw-Hill, New York, 2011, Chaps. 5, pp.411-450.

13 Yilmaz, T.O. and Rockwell, D., "Flow Structure on Finite-Span Wings due to Pitch-Up Motion", Journal of Fluid Mechanics, Vol. 691, 2012, pp518-545.

14 Visbal, M.R., "Three-Dimensional Flow Structure on a Heaving Low-Aspect-Ratio Wing", 49th AIAA Aerospace Sciences Meeting including the New Horizons Forum and Aerospace Exposition, AIAA 2011-219. 